\title{
Changes in the retreatment radiation tolerance of the spinal cord with time after the initial treatment
}

Thomas E Woolley ${ }^{1}$, Juan Belmonte-Beitia ${ }^{2}$, Gabriel F Calvo ${ }^{2}$, John W Hopewell ${ }^{3}$, Eamonn A Gaffney ${ }^{4}$ and Bleddyn Jones ${ }^{5}$

${ }^{1}$ Cardiff School of Mathematics, Cardiff University, UK

${ }^{2}$ Department of Mathematics, University of Castilla-La Mancha, Ciudad Real, Spain

${ }^{3}$ Green Templeton College, University of Oxford, UK

${ }^{4}$ Wolfson Centre for Mathematical Biology, Mathematical Institute, University of Oxford, UK

${ }^{5}$ Gray Laboratory, CRUK/MRC Oxford Institute for Radiation Oncology, University of Oxford, UK

Key words: radiotherapy, re-treatment, CNS tolerance, spinal cord, mathematical modelling, myelopathy

Running Title: Spinal cord retreatment tolerance

Author for correspondence:

Professor John W Hopewell

Green Templeton College

University of Oxford

Woodstock Road

Oxford OX2 6HG, UK

e-mail: john.hopewell@gtc.ox.ac.uk 


\section{Abstract}

Purpose: To estimate, from experimental data, the retreatment radiation 'tolerances' of the spinal cord at different times after initial treatment.

Materials and methods: A model was developed to show the relationship between the biological effective doses (BED) for two separate courses of treatment with the BED of each course being expressed as a percentage of the designated 'retreatment tolerance' BED value, denoted $B E D_{1}$ and $B E D_{2}$. The primate data of Ang et al (2001) was used to determine the fitted parameters. However, based on rodent data, recovery was assumed to commence 70 days after the first course was complete, and with a non-linear relationship to the magnitude of the initial BED $\left(B E D_{\text {init }}\right)$.

Results: The model, taking into account the above processes, provides estimates of the retreatment tolerance dose after different times. Extrapolations from the experimental data can provide conservative estimates for the clinic, with a lower acceptable myelopathy incidence. Care must be taken to convert the predicted $B E D_{2}$ value into a formal BED value and then a practical dose fractionation schedule.

Conclusions: Used with caution, the proposed model allows estimations of retreatment doses with elapsed times ranging from 70 days up to three years after the initial course of treatment.

\section{NOTE TO REVIEWERS}

A Graphic User Interface is provided with this manuscript to aid calculation based on the proposed model. This can be downloaded as a 32 or 64 bit version using the following link https://www.dropbox.com/sh/5qf5f2us0shb3ex/AAB65t768H_0RWrsmslzYSOna?dl=0 where additional instruction can also be obtained 


\section{Introduction}

Radiotherapy retreatment has been shown to be useful in many clinic situations, for highly selected patients with recurrent local disease and where other treatment options are limited (Medin et al., 2012, Jones and Grant 2014). Two recent publications have proposed methods for estimating the change in spinal cord radiation tolerance with increasing elapsed time between the initial radiotherapy and retreatment (Jones and Grant, 2014, Jones and Hopewell, 2014).

These models rely on the basic Linear-Quadratic (LQ) concept of biologically effective dose (BED), which is the maximum possible dose required to achieve a specified biological effect when given in a large number of small dose fractions, $n$, of equal size, $d$, giving a total dose, $D=n d$. The equation defining this relationship is given by

$$
B E D=n d\left(1+\frac{d}{\alpha / \beta}\right)
$$

where the $\alpha / \beta$ ratio is a constant which represents the tissue specific biological parameter that controls the tissue sensitivity to the radiation dose, n.d (Jones et al., 2001). Moreover, working with BED is preferable to the use of physical doses, since BED accommodates for changes in and fraction size $(d)$ and number $(n)$ in the estimation of biological iso-effects. Estimates of the change in spinal cord radiation tolerance, with increasing elapsed time between the initial radiotherapy and retreatment, requires a reasonable characterization of the relationship between the BED of the initial treatment course $\left(B E D_{\text {init }}\right)$, relative to the designated BED for 're-treatment tolerance', and the elapsed time $(t)$ between the two treatments, while maintaining a constant $\alpha / \beta$ ratio, e.g. 2 Gy for spinal cord tissue.

The previous study by Jones and Hopewell (2014) used an analysis based on the primate study by Ang et al (2001), in which an initial fixed dose was followed by retreatment at time 
intervals of one, two and three years, with the optional use of conservative parameters for human application. This model may not be reliable for retreatment time intervals of between three months to one year, although it was compatible with the results of several studies in rodents which used variable intial treatment doses and variable retreatment time intervals (4-6 months), as summarized by Jones and Hopewell (2014). Consequently, there is a need to investigate the change in tolerance for re-treatment intervals of less than one year, while also taking into account observations that

- spinal cord recovery after the initial radiation dose only begins around 70 days later (Wong and Hao, 1997; van der Kogel, 1997)

- initially, the rate of recovery appears to depend on the value of $B E D_{\text {init }}$, but is considerable after higher initial doses. The rodent experiments show little or no recovery around six months after initial BED values that were between $20-30 \%$ of the full tolerance BED value. It is also possible that later recovery can occur in more long-lived animal species, as is assumed below.

These conditions imply that the degree of recovery varies with time and depends on the initial dose given. Faster recovery occurs after high initial doses, presumably due to activation of compensatory mechanisms that are a response to the radiation insult. Delayed recovery after lower initial doses is considered more likely to be associated with that of normal tissues with a slow cell turnover time (144 days in rodent spinal cord - Hornsey et al., 1981; Zeman et al., 1964). Such a slow natural tissue turnover would restrict recovery to occur over a much longer time frame. This broad assumption is consistent with clinical experience, since severe retreatment reactions do not appear to be problematic in the 'low dose' regions of the central nervous system (CNS) situated away from the initial course high dose target volume. 
The primary objective of the present study is to extend the approach of Jones and Hopewell (2014), which expressed the initial and retreatment BED as a percentage of the 'tolerance' $\mathrm{BED}$ for each of the two treatments (i.e. $B E D_{1} \%$ and $B E D_{2} \%$ ), by providing more clinically relevant fits of the primate data and secondly to also explicitly take into account the implications of the rodent data. This will include the delayed onset of rapid recovery after high initial doses, as well as more delayed and slower recovery after low initial doses. Finally, incorporating a refined conservative approach, based on the comparison of the doseresponse curves for myelopathy in humans and rhesus monkey and with the help of worked examples, the experimental data on retreatment is extrapolated into a clinical setting with allowances for different clinical circumstances which may influence tissue tolerance. These objectives are also facilitated by a Graphical User Interface (GUI) containing the model, which allows for the rapid investigation of model predictions.

\section{Methods}

\subsection{Experimental data sets}

The data sets are essentially the same as those used in the previous publication by Jones and Hopewell (2014), where further details are provided about the reasons for selecting these data on the basis of anatomical position and the nature of the neurological injury. In addition, the dose-response data from rhesus monkey are compared with a previously unpublished human dose-response curve for myelopathy (Ang, personal communication 1998) in order to support the conservative extrapolation of the rhesus monkey findings into a clinical setting, as detailed below. 


\subsection{Description of mathematical model: The relationship between $B E D_{1} \%$ and $B E D_{2} \%$}

\subsubsection{Aspects of the initial treatment tolerance dose}

The concept of 'tolerance dose' implies a radiation dose, after which, there is an unacceptable probability of a specific complication. In animal experiments, in order to have reasonable numbers of events for statistical analysis, probability levels such as 0.1 or 0.5 (10 and $50 \%$ incidence levels, respectively) have been used as end points. On the other hand, for human patients, 'tolerance dose' can imply a dose and fractionation schedule that is close to the threshold for the development of a specific adverse radiation induced reaction. Usually the probability of this adserse reaction or side effect is kept very low, which is very important for the spinal cord where the complication is radiation myelopathy, resulting in paralysis. Thus, the concept of 'tolerance doses' differ between animal experiments and human patients; consequently, an important feature of this study is the accommodation of these conceptual and quantitative differences by extrapolation to the levels of incidence acceptable in humans from the original animal experimental data, which use higher incidence probability levels than would be accepted in the clinic. This is further considered in section 2.3.

In practice, the tolerance dose for the human spinal cord is the maximum dose permitted to be given to the spinal cord by the physician, based on the assessment of additional risk factors. Modern radiotherapy treatment planning systems can incorporate this limit as an important constraint. While this is often the guiding constraint, higher doses are permitted in some circumstances for very small volumes of spinal tissue (Kirkpatrick et al. 2010). For human spinal cord (Schultheiss et al. 2008), the typically accepted human tolerance dose for a primary treatment is $50 \mathrm{~Gy}$ in 25 fractions of $2 \mathrm{~Gy}$, which is defined as $D_{T O L}$ and is a nonconservative treatment policy. This constitutes a $\mathrm{BED}$ value $\left(B E D_{T O L}\right)$ of $100 \mathrm{~Gy}_{2}$, the subscript referring to the standard 2 Gy $\alpha / \beta$ ratio for spinal cord. This BED value normally applies to fit patients with no adverse risk factors associated with age, significant previous 
chemotherapy, surgery and other concomitant medical conditions that may reduce this tolerance dose. Patients with high risk factors must be carefully considered by the responsible clinician, who may adopt a conservative policy, for example by adjusting the total dose down to deliver a lower BED value such as 88 or $92 \mathrm{~Gy}_{2}$ for schedules of $44 \mathrm{~Gy}$ or $46 \mathrm{~Gy}$, using 2 Gy fractions, respectively. On average, this represents around a 10\% reduction in BED to maintain tolerance, then defined as a similar risk of myelopathy in high-risk patients as would occur with the non-conservative policy in standard patients. Also, care must be taken when the dose per fraction is changed to produce, for example, a BED of $85.5 \mathrm{~Gy}_{2}$ using a schedule of $45 \mathrm{~Gy}$ in twenty-five fractions of $1.8 \mathrm{~Gy}$, or a BED of $96 \mathrm{~Gy} 2$ when using $24 \mathrm{~Gy}$ in four fractions of 6 Gy. In special situations an 'ultra-conservative' approach might be needed when the dose representing tolerance is reduced further, for example by around $20 \%$, to a BED of $80 \mathrm{~Gy}_{2}$, which is equivalent to a dose of $40 \mathrm{~Gy}$ in 20 fractions of 2 Gy fractions produces.

In a clinical retreatment situation, the concept of a BED 'tolerance' value for the human spinal cord may need to change particularly if an initial dose equal to or near tolerance has been delivered. This will require a reassessment of the acceptable risk of myelopathy on retreatment. In general terms this is designated $B E D_{R \%}$, where $R$ is the percentatage incidence of risk of myelopathy considered clinically acceptable in a particular retreatment situation. This represents a change from the original publication (Jones and Hopewell 2014) where $B E D_{T O L}$ was rather loosely used to represent acceptable 'tolerance' on retreatment. For the purpose of the present discussion this 'acceptable' risk in a retreatment situation has been taken as a nominal $R=1 \%$ incidence of myelopathy on retreatment and thus $B E D_{R \%}=$ $B E D_{1 \%}$. By default, $B E D_{R \%}$ is higher than $B E D_{T O L}$ in a primary treatment, where the acceptable risk of myelopathy is normally very much lower (as discussed below). The $B E D_{R \%}$ value is used to define the scale for both the percentage tolerance BED of the initial 
treatment $\left(B E D_{1}\right)$, and the estimated maximally tolerated retreatment percentage tolerance $\left(B E D_{2}\right)$. It is important to note that both $B E D_{1}$ and $B E D_{2}$ are non-dimensional and are formally given in terms of the initial $\mathrm{BED}\left(B E D_{\text {init }}\right)$ and the estimated retreatment dose $\mathrm{BED}$ $\left(B E D_{\text {ret }}\right)$, both of which are divided by the same retreatment 'tolerance', $B E D_{R \%}$. Explicitly,

$$
\text { First Treatment } B E D_{1}=\frac{B E D_{\text {init }}}{B E D_{R \%}} \times 100 \%,(1 a)
$$

$$
\text { Second Treatment } B E D_{2}=\frac{B E D_{r e t}}{B E D_{R \%}} \times 100 \% \cdot(1 b)
$$

If a different risk of myelopathy was to be considered acceptable in a given clinical retreatment situation then the 'acceptable risk' needs to be defined and the presently used BED, assumed to carry a $1 \%$ risk $\left(B E D_{1 \%}\right)$, replaced by the selected risk value, $B E D_{R \%}$. For clarity, the definitions of the above parameters and other variables used in the model are summarised in Table 1.

Working initially with BED is preferable to the use of physical doses since BED values incorportate fractionation effects. Moreover, using ratios of BED values, namely $B E D_{1}$ and $B E D_{2}$ for the initial and retreatment doses, greatly simplifies translating data from the rhesus monkey and other species to the human clinical setting.

\subsubsection{Finding the relationship between $B E D_{1}$ and $B E D_{2}$}

A relationship is required between $B E D_{1}$ and $B E D_{2}$, that accommodates all the radiobiological retreatment requirements for the spinal cord was proposed by Jones and Hopewell (2014). In a more simplified form this is represented by the equation

$$
B E D_{2}=100\left(1-\frac{B E D_{1}}{100}\right)^{\frac{1}{r(t)+1}}
$$


where $r(t)$ is the recovery function that depends on the elapsed time, $t$, from the initial treatment. This model assumed that $r(t)$ only increases with time from an initial value of zero, so that at time $t=0$

$$
B E D_{2}+B E D_{1}=100 \%
$$

the condition of no recovery. It also assumes that for sufficiently long elapse times, $B E D_{2}$ approaches $100 \%$, indicating full recovery from the initial dose and the retreatment dose reaches the level of tolerance.

However, the relationship proposed by Jones and Hopewell (2014) between $B E D_{2}$ and $B E D_{1}$, as represented by equation (2), did not consider that dymanic recovery could be dependent on the value of $B E D_{1}$, such that recovery did not occur as rapidly when $B E D_{1}<\overline{B E D}$, where $\overline{B E D}$ represents a percentage threshold of $B E D_{1 \%}\left(\right.$ or $B E D_{R \%}$ if the risk at retreatment is changed). This provides a boundary between the efficient recovery seen after higher $B E D_{1}$ values, where relatively rapid recovery may initially be seen, and after lower $B E D_{1}$ values where recovery is apparently much slower. Specifically, as mentioned in the introduction, rodent experiments show little recovery after initial $B E D_{1}$ values of up to $20-30 \%$ of in initial dose represented by $B E D_{1 \%}$. Hence, threshold values for $\overline{B E D}$ appear to be around $20-30 \%$. However, a $\overline{B E D}$ value of $35 \%$ is recommended for standard use. This value is precautionary, since it will apply delayed recovery at slightly higher $B E D_{1}$ values, thus avoiding unintended overdosage on re-treatment. Although this value was selected with the above safety considerations in mind, the sensitivity analysis given in Table 2 and through the GUI calculator provided (see Appendix B), provide additional reassurance that this choice does not lead to signficant disadvantages. Also, a large number of clinical problems deal with $B E D_{1}$ values greater than $\overline{B E D}$, when the results obtained for $B E D_{2}$ are largely independent of the selected value of $\overline{B E D}$. 
To accommodate these constraints, equation (1) is generalised to

$$
B E D_{2}=100\left(1-\frac{B E D_{1}}{100}\right)\left[1+\left(\left(1-\frac{B E D_{1}}{100}\right)^{\frac{-r(t)}{r(t)+1}}-1\right) f\left(B E D_{1}, r(t)\right)\right]
$$

where the function $f\left(\mathrm{BED}_{1}, r(t)\right)$ in Eq. (3) is given by

$$
f\left(B E D_{1}, r(t)\right)=\frac{1}{2}\left[1+\tanh \left[s_{0}\left(B E D_{1}-\frac{\overline{B E D}}{1+s_{1} \cdot r(t)}\right)\right]\right],
$$

which has values between zero and unity. In particular, $f\left(B E D_{1}, r(t)\right)$ has been constructed to control the transition between the initial BED-dependent early and later recovery, as determined by the controlling parameters $s_{0}, s_{1}$, and $\overline{B E D}$, with the latter indicating where the transition occurs between early and later recovery. Hence, $f\left(B E D_{1}, r(t)\right) \approx 1$ corresponds to the early recovery of Jones and Hopewell (2014), whilst $f\left(B E D_{1}, r(t)\right) \approx 0$ indicates essentially no recovery. Further justification for the functional form of $f\left(B E D_{1}, r(t)\right)$ is provided in Appendix A.

\subsubsection{Estimation of the parameters $s_{0}, s_{1}$ and $\overline{B E D}$}

These three parameters are a novel addition to the model introduced by Jones and Hopewell (2014). It takes account of the behaviour of $B E D_{2}$ in relation to low values of $B E D_{1}$ observed in the rodent data (see Figure 3), which this earlier model did not include.

Specifically, $\overline{B E D}$, acts as a boundary between the slow and more rapid recovery regions, which depend on the value of $B E D_{1}$. The basic concept that a recovery process may not be initiated unless a specific level of damage is reached is supported by the results of a number of studies in both acute and late responding normal tissues. For example, in the epidermis the accelerated repopulation of basal stem cells does not occur until the normal density declines by $\sim 50 \%$ a function of higher dose (Morris and Hopewell, 1988), while in the brain an 
abortive proliferation of endothelial cells, the primary target cells population for delayed white matter necrosis was only seen when the cells population size fell be $40 \%$ and the associated capillary density by $10 \%$ (Lyubimova and Hopewell, 2004).

The parameter $s_{0}$ is dimensionless and controls how vigorously the transition between later and earlier recovery occurs as the size of $B E D_{1}$ increases. Since this transition may be sharp, then $s_{0}$ needs to be sufficiently large. Because the scaling value of $B E D_{1}$ is up to $100 \%$, this simply requires that $100 s_{0} \gg 1$, and once $s_{0}$ is much larger than 0.01 the model predictions are expected to be insensitive to any further changes in its value. Furthermore, such values of $s_{0}$ are consistent with the available rodent data and, inpractice, a $s_{0}$ value of 0.15 , has been used.

Finally, the dimensionless parameter $s_{1}$ governs the timescale over which the low initial $B E D_{1}$ values influence the later recovery. If $s_{1}$ is sufficiently small, it will indicate the timescale of recovery of low $B E D_{1}$ values. In the subsequent calculations the of $s_{1}$ value of 0.1 was used since again it was consistent with the available data. In cases where the $B E D_{1}$ value is greater than $\overline{B E D}$, then none of the parameters $s_{0}, s_{1}$ and $\overline{B E D}$ will greatly influence the solution to the fit in the higher $B E D_{1}$ region. In a high proportion of clinical situations is likely that the initial treatment will place the patient in this high $B E D_{1}$ region on treqatment and it is expected that the results obtained for $B E D_{2}$ will be insensitive to variations in the values of these parameters (see Table 2 and Appendix B). Furthermore, because the results are insensitive to the value of these parameters (which were not obtained by precise fitting), the values were carefully selected to have minimal impact on the final result, namely the value of $B E D_{2}$. However, since a Graphical User Interface (GUI) calculator is included with this publication for the general user, that user is allowed to alter the values of $s_{0}, s_{1}$ and $\overline{B E D}$ so that they may check the assumption that changing these values do not impact on the outcome. 
In retreatment situations where $B E D_{1}$ is less than or close to $\overline{B E D}$, no larges changes in $B E D_{2}$ are produced if $s_{0}$ and $s_{1}$ are varied within a range of $+/-40 \%$. However, since only very limited data are available for low $B E D_{1}$ values, a conservative approach is now recommended in the revised model with respect to the values of $s_{0}$ and $s_{1}$ used. This is not a particular clinical limitation, since in any event $B E D_{2}$ will be reasonably high and likely to produce clinical benefits. Overall, the available rodent data are sufficiently large to exclude overfitting for a three-parameter model. Moreover, the model does not suffer from one of the consequences of overfitting (where small changes in the parameter values would result in large output changes). Furthermore, these parameters can be readily adjusted in the GUI linked to this publication, in order to confirm the minor impact of these parameter values on the $B E D_{2}$ output.

The translation of rodent radiation toxicity data to that of the rhesus monkey and ultimately to humans would also appear to be totally justified since the time of onset of myelopathy related to selective white matter necrosis and that the reaction kinetics are similar in rodents (Hopewell et al., 1987) and the rhesus monkey and human (Ang et al., 2012).

\subsection{Determining $r(t)$. Part 1. Extrapolating from experimental to a clinical level of incidence}

Due to the absence of appropriate human data, the data from Ang et al (2001) for radiation myelopathy in the rhesus monkey was used in this study. The recovery function, $r(t)$, is assumed to be the same for both rhesus monkey and human spinal cord. The calculations 
below provide the maximum possible re-treatment doses that could be safely used for retreatment of the spinal cord (as discussed in Jones and Hopewell 2014).

In the experimental study by Ang et al (2001), different treatments were compared at $E D_{10}$, the dose associated with a $10 \%$ incidence of myelopathy. However, to increase the clinical relevance of this data in the subsequent calculations, the dose associated with a $1 \%$ ( $p=$ 0.01 ) incidence of myelopathy was determined by extrapolation. This level of incidence might be acceptable in a retreatment situation where the risk of developing paralysis, or other fatal developments, from tumour progression may be even higher. However, there will be other factors that may require a lower incidence, and should be determined on a patient by patient basis.

In order to determine $r(t)$, which relates $B E D_{1}$ and $B E D_{2}$, it is necessary to consider the extrapolation between experimental levels of myelopathy incidence obtained in experimental animals to the lower incidence range that would be acceptable in the clinic.

The study in the rhesus monkey considered the variation in the incidence of myelopathy, with total dose, for re-irradiation at 1, 2 and 3 years, after a fixed initial dose of 44 Gy given in 2.2 Gy fractions (a BED of $92.4 \mathrm{~Gy}_{2}$ ). These were compared with a dose response curve for a single course of irradiation using 2.2 Gy fractions. The resulting dose-effect curves are shown in Figure 1(a), fitted by the probit model, relating the probability of myelopathy to total dose, Probability of myelopathy at dose $D=P\left(D, D_{50}, \gamma_{50}\right)=\frac{1}{\sqrt{2 \pi}} \int_{-\infty}^{\gamma_{50}\left(\frac{D}{D_{50}}-1\right)} \exp \left(-u^{2} / 2\right) d u,(5)$ where $u$ is the dummy integration variable, $D_{50}$ is the dose corresponding to the $50 \%$ incidence level and $\gamma_{50}$ is a measure of the steepness of the dose-response curve, which satisfies

$$
\gamma_{50}=\sqrt{2 \pi} \cdot m_{50} \cdot D_{50}
$$


where $m_{50}$ is the gradient of the dose-response curve at the $50 \%$ incidence level.

An assumption made in the curve fitting presented in Figure 1(a) is that $m_{50}$ is fixed for all curves (both human and monkey), i.e. $\gamma_{50} / D_{50}$ is fixed. Thus, the value of $\gamma_{50} / D_{50}$ was estimated from the single course dose-effect data of Ang et al (2001). Subsequently, all curves are now parallel and the fitting of all other curves (for retreatment at the different elapsed times) only has one degree of freedom, namely, the curves are shifted left, or right, relative to one another. Although only one data point is, thus, needed to determine the lateral shift of these curves, in practice the lateral shift fit was actually performed using all available data points using a least squares fit, with $\gamma_{50} / D_{50}$ fixed as stated above.

The single course treatment probit distribution shown in Figure 1(a), combined with the fitted values for $\gamma_{50} / D_{50}$, and $\gamma_{50}$, can be used to convert the probability of myelopathy to dose for the irradiation of the spinal cord and, in particular, allows extrapolation from the Ang et al (2001) estimates, based on the re-irradiation $E D_{10}$, (a $10 \%$ incidence or $\left.p=0.1\right)$ to the lower value of $E D_{1}$ (a $1 \%$ incidence or $p=0.01$ ) as indicated in Figure 1(b).

\subsection{Determining the recovery function, $r(t)$. Part 2. Determining $r(t)$ using the data from} the rhesus monkey.

\subsubsection{Re-irradiation iso-effective doses}

The probit model allows the determination of the maximum re-irradiation doses for the rhesus monkey that are associated with a designated probability of myelopathy after an elapsed time of $t=1$ year, $t=2$ years and $t=3$ years following the initial irradiation dose of $44 \mathrm{~Gy}$ in 20 fractions. As mentioned previously a nominal 1\% risk of myelopathy ( $p=$ 0.01 ) is used in this manuscript for illustrative purposes, but noting there is little change in the resulting curve if this is increased (see Figure 3). 
The resulting retreatment doses are denoted by $D_{\text {ret }}(t)$, and can be evaluated using the three re-irradiation probit curves, shown in Figure 1. For an incidence level of $p=0.01$ the values are

$$
D_{\text {ret }}(1)=48.7 \mathrm{~Gy}, D_{\text {ret }}(2)=56.6 \mathrm{~Gy} \text { and } D_{\text {ret }}(3)=59.3 \mathrm{~Gy} \text {, }
$$

at 1, 2 and 3 years, respectively as could also be inferred from inspection of Fig. 1b.

Following a primary irradiation, the associated dose $\left(D_{1 \%}\right)$ is 60.9 Gy for the same level of incidence $(p=0.01)$.

\subsubsection{Converting the tolerance doses for the rhesus monkey into biologically effective doses}

The basic BED equation can be used to convert the above $1 \%$ risk of myelopathy doses into the biologically effective dose by using

$$
B E D_{1 \%}=D_{1 \%}\left(1+\frac{d}{\alpha / \beta}\right)
$$

where $d$ is $2.2 \mathrm{~Gy}$, and $\alpha / \beta=2 \mathrm{~Gy}$ in the study by Ang et al (2001). Hence, for the $D_{1 \%}$ the rhesus monkey re-irradiation 'tolerance' biologically effective dose with incidence level of $p=0.01$ is

$$
B E D_{1 \%}=60.9\left(1+\frac{2.2}{2.0}\right) \mathrm{Gy}_{2}=127.9 \mathrm{~Gy}_{2}
$$

Similarly, the maximum retreatment biologically effective dose at 1 year, denoted by $B E D_{\text {ret }}(1)$, for the same level of incidence, is given by equations (1) and (6) such that:

$$
\begin{aligned}
B E D_{2}(1)= & \frac{B E D_{r e t}(1)}{B E D_{1 \%}} \cdot 100 \%=\frac{D_{r e t}(1)\left(1+\frac{d}{\alpha / \beta}\right)}{127.9 \mathrm{~Gy}_{2}} \cdot 100 \%=\frac{48.7\left(1+\frac{2.2}{2.0}\right)}{127.9 \mathrm{~Gy}_{2}} \cdot 100 \\
& =80.0 \%,(8 a)
\end{aligned}
$$

and analogously $B E D_{2}(2)=92.9 \%$ and $B E D_{2}(3)=97.4 \%$. 
In addition, using the initial dose $\left(B E D_{\text {init }}\right)$ of $44 \mathrm{~Gy}$, the initial percentage tolerance BED value $\left(B E D_{1}\right)$ for the rhesus monkey is given by

$$
B E D_{1}=\frac{B E D_{\text {init }}}{B E D_{1 \%}} \cdot 100 \%=\frac{44\left(1+\frac{2.2}{2.0}\right)}{127.9} \cdot 100 \%=72.2 \% .(8 b)
$$

A close examination at equations (7) and (8) shows that the BED relative effectiveness factor,

$$
\left(1+\frac{d}{\alpha / \beta}\right)
$$

is cancelled between the numerator and denominator, providing that $d$ remains the same for both treatment courses. Thus, the fact that Rhesus monkey experiments used fractions of 2.2Gy, whereas clinical fractions of $2 \mathrm{~Gy}$ for human are commonplace, does not impact on the determination of the recovery function, $r(t)$, for rhesus monkey, or the assumption that the method can be used for humans, due to this cancellation.

\subsubsection{Converting biologically effective dose into values of $r(t)$ for the rhesus monkey}

The initial percentage biological effective dose $\left(B E D_{1}\right)$ and, via probit curves, the three maximal retreatment doses $B E D_{2}(1), B E D_{2}(2)$ and $B E D_{2}(3)$ for a myelopathy incidence of $p=0.01$ for the rhesus monkey enables the calculation of $r(1), r(2)$ and $r(3)$ using equations (3) and (4). With the estimates of the parameters $s_{0}=0.15, s_{1}=0.1$ and $\overline{B E D}=$ $35 \%$, from section 2.2.3, a numerical root-finding algorithm such as the Newton-Raphson procedure (Burden et al 2011), can be used to find

$$
r(1)=4.75, r(2)=16.4 \text { and } r(3)=48.0
$$

These $r(t)$ estimates can be calculated automatically by the Graphical User Interface (GUI) supplied with this paper. 
In addition, recovery is initially delayed for 70 days, corresponding to an Initial Recovery Onset time of $t_{I R O}=70 / 365=0.19$ years. This is in agreement with the available literature (Wong and Hao, 1997; van der Kogel 1997). Hence $r(t)=0$ for $t \leq t_{I R O}$. Consequently, a cubic polynomial interpolation of $r(t)$ for $t \geq t_{I R O}$ can be used, whereby

$$
r(t)=\left\{\begin{array}{c}
0, t \in\left[0, t_{I R O}\right] \\
a+b t+c t^{2}+d t^{3}, t \in\left[t_{I R O}, 3\right]
\end{array}\right.
$$

for $t$ in units of years.

Fixing $r\left(t_{I R O}\right)=0$ and with the values of $r(1), r(2)$ and $r(3)$, there are four constraints for the four unknowns, $a, b, c$ and $d$, which can be evaluated using the equations summarised in Appendix $\mathrm{C}$ to give

$$
a=-1.44, b=8.28, c=-4.51 \text { and } d=2.41 \text {. }
$$

The recovery function, calculated using Equation (9), is plotted, together with the individual data points, in Figure 2 . The recovery function associated with $p=0.01$ does not vary very significantly with a change in the level of risk up to $p=0.1$ as illustrated in the insert in Figure 2.

Extrapolation outside the range of the experimental data can be subject to significant errors. However, it is reasonable, based on the present data set, to consider elapsed times, $r(t)$, of greater than 3 years, since by then recovery is almost complete, i.e. $B E D_{2}(t)$ at 3 years is almost $100 \%$. Thus, only relatively small errors will result as $r(t)$ values increase further since this simply shifts $B E D_{2}(t)$, as given by Eqns (3) and (4), closer to $100 \%$.

In summary, calculation of the recovery function, $r(t)$, via equation (9), using the data from the rhesus monkey of Ang et al (2001) and a probit model, allows extrapolation between experimentally used and clinical acceptable levels of incidence. This fully determines the relationship between the percentage maximum retreatment biologically effective dose, 
$B E D_{2}(t)$, and the initial percentage dose, $B E D_{1}$, via equations (3), (4) and (9). Subsequently it is illustrated how this information can be implemented for specific human, clinical, examples, including how conservatism can be incorporated, and how a GUI facilitates calculations.

\section{Results}

\subsection{The Basic Model Relating $B E D_{1}$ and $B E D_{2}$, based on Rhesus Monkey Data}

In any primary clinical treatment, the chosen radiation tolerance of the spinal cord is normally associated with a very low probability of patient myelopathy. However, in a retreatment situation, different criteria might be adopted, where a higher myelopathy risk might be considered clinically acceptable. For illustrative purposes in the present calculations, specifically involving human retreatment, a probability of $p=0.01$ (a $1 \%$ risk of myelopathy) has been nominally used for illustrative purposes on the assumption that to withhold retreatment could be associated with even greater risks. The basic model considered is based on this assumption, and also that rhesus monkey data can be used to estimate the recovery function, $r(t)$, in the human.

Initially, in the absence of any clinical conservatism, the resulting relationship between the percentages of $B E D_{2}$ and $B E D_{1}$, as outlined previously, from the experimental Rhesus monkey data is plotted in Figure 3a for a myelopathy risk probability of $p=0.01$, that is $\mathrm{R}=$ 1\%. For comparison, a plot was also made (Figure $3 b$ ) for a higher risk of myelopathy, $p=$ $0.1(\mathrm{R}=10 \%)$, as initially used by Ang et al (2001).

The curves in Figure 3 show how the retreatment dose is predicted by both the elapsed time from, and the size of, the initial treatment dose, expressed as percentages, $\mathrm{BED}_{1}$ of the biologically effective tolerance dose. The dashed straight diagonal curve represents the 
situation where there would be no recovery. Thus, for the low dose region, $\mathrm{BED}_{1}<\overline{\mathrm{BED}}=$ $35 \%$, the relationship between $\mathrm{BED}_{1}$ and $\mathrm{BED}_{2}$ tracks this "no recovery" diagonal line when the time to retreatment is short. Beyond this point, at least initially, stimulated recovery occurs. Due to the discrepancy between these faster and slower recovery rates a transition region is needed in the model. This transition region creates the observed dip linking the slow and fast recovery regions. These curves provide a more biologically appropriate fitting than that obtained by Jones and Hopewell (2014), especially when low initially $\mathrm{BED}_{1}$ values were given which do not appear to be associated with any recovery for up to around 6 months.

The fitted curves for 1,2 and 3 years of elapsed time pass through the rhesus monkey data points. Furthermore, the ability to consider smaller elapsed times allows comparisons with previously published experimental rodent data, which indicated the probable existence of a region where no short term recovery was observable, while retaining the possibility of later recovery in longer lived animals. Such short timescales are presented in Figure 3, where rodent data results are superimposed on the percentage retreatment BED recovery curves for elapsed times between 4, 5 and 6 months. The dependency of transition in recovery with the size of the initial BED can be seen in both the rodent data and the predicted curves at a qualitative level. However, quantitative comparisons are not truly appropriate given that the tolerated doses in rodent was based on a myelopathy incidence level of 50\%. Finally, from a comparison of figures $3 \mathrm{a}$ and $3 \mathrm{~b}$ it can be seen that predictions are very similar for a probability of myelopathy at the $p=0.01$ and 0.1 level, reflecting that there is only a small change in the recovery function with this order of magnitude change in myelopathy probability.

Before proceeding to worked examples, it is important to consider the addition of a reasonable degree of clinical conservatism in the modelling. 


\subsection{Comparison of human and rhesus monkey dose-effect data for myelopathy and the need for conservatism and ultra-conservatism}

As defined in equations (1a) and (1b), $B E D_{1}$ and $B E D_{2}$ are defined as ratios of the maximally permitted $B E D_{R \%}$ (nominally $B E D_{1 \%}$ for the present illustrative purposes) but recognising that this may need to vary from patient to patient. For a specified elapse time $(\mathrm{t}), B E D_{2}$, is derived from Figure 3, and included in Equation (1b) to determine the absolute clinical human $B E D_{\text {ret }}$ value for the subsequent clinical treatment schedule, which may be specified by dose/fraction or the number of treatment fractions.

Currently, there is relatively limited data available for the human spinal cord, for equivalent 2 Gy/fraction doses. However, some human data (Ang: personal communication, 1996) has been compared with the data for the rhesus monkey in Figure 4(a). In this analysis, again in order to be conservative, the human dose-effect relationship is fitted to all the human data points except for that at the highest dose, using the same fitting procedure as described for the rhesus monkey, namely this human dose-effect curve is, by construction, a shift of the rhesus monkey dose-effect curve along the dose axis. The resulting $p=0.01$ ( $1 \%$ incidence of myelopathy) dose is $54.8 \mathrm{~Gy}$. This is consistent with the value quoted in the 2010 Quantec publication (Kirkpatrick et al., 2010) where a dose to the spinal cord of $54 \mathrm{~Gy}$, in $2 \mathrm{~Gy}$ fractions, was said to be associated with less than a $1 \%$ risk of myelopathy. This forms the basis of the non-conservative model developed above. Extrapolation of the curve for human myelopathy data (Figure 4a) indicated that the dose associated with 'tolerance' in human spinal cord, 50 Gy in 25 fractions of 2 Gy (van der Kogel, 1989), is associated with a very low risk of myelopathy, $0.12 \%(p=0.0012)$ using the present analysis.

Although a degree of caution was adopted in the above analysis by removing a potential radio-resistant outlier from the human curve (Figure 4(a)) the limited data also means that statistical hypothesis testing is not feasible. Hence, there is a need for cautious conservatism 
in the approach, as used in clinical practice, even with respect to patients where there was, in the initial treatment, no specific factors that might have indicated the need for a more conservative or even an ultra-conservative approach. Further conservatism is often required when additional risk factors exist in some patients. In such compromised patients, the radiation dose is conventionally reduced as indicated previously to preserve iso-effective treatments.

This conservatism can be accommodated within the proposed framework and could simply be taken in the form of either a $10 \%$ or $20 \%$ reduction in the above dose of $54.8 \mathrm{~Gy}$, with these reduced doses still being taken, for illustrative purposes, as being associated with a $1 \%$ incidence of myelopathy. A comparable approach could be adopted for the doses associated with different risks of the incidence of myelopathy that could be considered acceptable in a retreatment situation.

However, an alternative more constant approach, for a compromised patient with additional risk, would be to shift all probit curves to the left, by $C \%$. This entails initially finding the dose, $D_{1}$, corresponding to $1 \%$, i.e.,

$$
0.01=P\left(D_{1}, D_{50}, \gamma_{50}\right)
$$

where $P$ is the probit curve of dose response, for varying $D_{1}$, with parameters $D_{50}$ and $\gamma_{50}$ fixed. Then the curves are all shifted by a factor $S$ such that a probability of $1 \%$ now corresponds and to a dose of $D_{1}(1-C / 100)$, i.e.,

$$
0.01=P\left(S+D_{1}(1-C / 100), D_{50}, \gamma_{50}\right) .
$$

Thereafter the probit curve should include the computed shift, $P\left(S+D, D_{50}, \gamma_{50}\right)$, in order to calculate the relationship between dose and the probability incidence of myelopathy. Notably, since $D_{50}$ and $\gamma_{50}$ are unchanged, the shifted curves are all parallel. Critically, the effect of this shift is to cause the same probability of incidence to correspond to a lower dose. For the 
purpose of the present calculations $C=10 \%$ is illustrated in Figure 4(b). Hence, the recovery function is determined via a similarly compromised monkey with the same modelled prediction of the risk of myelopathy. The graphical user interface (GUI) provided with this paper allows the establishment of predictions with or without a conservative shift of $C \%$, for the retreatment dose-response curves to accommodate a compromised patient and a specified level of incidence, $p$ and, hence, further illustrative calculations beyond those of the worked examples are not required ${ }^{1}$.

In summary, the model presented here effectively allows either an upward or downward change in risk allocation according to the prevailing retreatment circumstances. With the $1 \%$ risk of myelopathy in humans $(p=0.01)$ normalised in a non-conservative approach to 54.8 Gy, the doses associated with different levels of risk, between $0.25 \%$ and $10 \%$ $(p=0.0025-0.1)$ are given in Table 2 . The BED values associated with this range of risk are also given for the non-conservative approach, along with the BED values associated with a conservative and ultra-conservative approach using either a simple 10 or $20 \%$ reduction in the values associated with different levels of risk in the non-conservative situation, or a comparable left shift of the original dose-effect curve. The changes associated with these different approaches are small.

Also, there will be occasional clinical situations where the initial treatment was given without any degree of conservatism because the patient was not considered to have any additional risk factors (i.e. treated with standard doses up to a BED of $100 \mathrm{~Gy}_{2}$ ), but that it is decided to apply a more conservative approach re-treatment (because of intervening chemotherapy, surgery, development of other medical conditions etc.). Then, it is important to understand

\footnotetext{
${ }^{1}$ Although the application of conservatism depends on a chosen risk level of myelopathy (with the illustrative risk level being taken to be 1\%), this dependency has little influence on the BED calculation. In particular, for low incidences of risk of myelopathy and low levels of conservatism it makes very little difference at which level of risk conservatism is applied, see Appendix D.
} 
that the GUI only operates with both initial treatment and re-treatment being at the same risk or BED level. In such patients, it is first necessary to set the non-conservative treatment risk or BED and calculate the retreatment dose $\left(B E D_{\text {ret }}\right)$ for the same risk. Then apply a conservative reduction to the estimated retreatment BED, e.g. reducing it by say $10-20 \%$, and then recalculate the reduced dose per fraction.

\subsection{Using the model relating $B E D_{1}$ and $B E D_{2}$, based on the animal data, for retreatment calculations in a clinical context, with an illustration of the graphical user interface $(G U I)$}

\subsubsection{Worked example 1: A patient's spinal cord initially received $40 \mathrm{~Gy}$ in 20 fractions,}

which in non-conservative cases is a dose significantly below that usually associated with the accepted spinal cord 'tolerance dose' (50 Gy in 25 fractions of 2 Gy). Further radiotherapy was required 2 years later. Determine the predicted safe retreatment 'tolerance' dose at retreatment, using $\alpha / \beta=2$ Gy for spinal cord tissue and with

(i) the use of a non-conservative model with 'tolerance' at retreatment still being accepted as 50 Gy in 25 fractions (with estimated incidence of myelopathy of $R=0.12 \% ; p=0.0012$ ) using the current model i.e. no higher risk of myelopathy is considered clinically acceptable;

(ii) the same conditions as in (i) above, but now in a patient with additional risk factors who can withstand less radiation for the same incidence of incidence of myelopathy. In a conservative situation this is judged to be about $10 \%$ reduction in BED compared with a patient who is not compromised,

(iii) the use of the non-conservative model but with a dose of $54.8 \mathrm{~Gy}$, associated with a $1 \%$ incidence of myelopathy ( $p=0.01$ ), now being considered as clinically acceptable at re-treatment.

\section{Solution}


In these calculations accuracy is restricted to at most three significant figures, so that a discrepancy with the GUI at this level of accuracy is simply a rounding error, which is much smaller than the accuracy of the model precision.

(i) With individual dose fractions of $2 \mathrm{~Gy}$, and $\alpha / \beta=2 \mathrm{~Gy}$ for human spinal cord the 'tolerance' associated with a dose of $50 \mathrm{~Gy}, B E D_{T O L}$ and the initial biologically effective dose $B E D_{\text {init }}$ are given by:

$$
B E D_{0.12 \%}=B E D_{T o l}=50\left(1+\frac{2}{2}\right)=100 G y_{2} \text { and } B E D_{\text {init }}=40\left(1+\frac{2}{2}\right)=80 G y_{2} .
$$

Thus, the percentage initial biologically effective dose, $B E D_{1}$ is given by the equation

$$
B E D_{1}=100 \times B E D_{\text {init }} / B E D_{\text {Tol }}=80 \%
$$

Given the curve fitting of the model and its parameter estimates, the corresponding value for the percentage retreatment biologically effective dose, $B E D_{2}(2)$, can read off from Figure 5 a. Using a non-conservative approach, the line in Figure 5a, generated from the intersection of $B E D_{1}=80 \%$ with the non-conservative 2 -year model prediction generates a predicted $B E D_{2}(2)$ value of $91.9 \%$. Since the nominal 'tolerance' level biologically effective dose $B E D_{0.12 \%}$ is $100 \mathrm{~Gy}_{2}$ the associated retreatment biologically effective dose is $91.9 \mathrm{~Gy}_{2}$ (see Figure 5a). Using this BED value, a suitable dose fractionation schedule can be tailored to this patient.

To retain consistent 2 Gy fractions the fraction number would involve rounding up or down to maintain fractionation discreteness. Rounding up would imply a $B E D_{\text {ret }}$ of $92 \mathrm{~Gy}_{2}$, a dose of 46 Gy delivered in 23 fractions of 2 Gy. However, rounding down would mean using a $B E D_{\text {ret }}$ of $88 \mathrm{~Gy}_{2}$ or a dose of $44 \mathrm{~Gy}$ in 22 fractions of $2 \mathrm{~Gy}$. It should be recognised that the small increase in dose produced by rounding up in this case would be associated with a very small increase in the level of predicted risk being accepted $(p=0.001205)$, while rounding 
down would clearly reduce the predicted risk $(p=0.000443)$, from what was seen as the acceptable risk of $0.12 \%(p=0.0012)$.

In a palliative situation further reductions in the number of dose fractions might be considered appropriate. For example, using a course of 12 equal dose fractions then the total retreatment dose $D$ would be

$$
91.9=D\left(1+\frac{\frac{D}{12}}{2}\right)
$$

The relevant solution for $D$ is $36.5 \mathrm{~Gy}$, the dose per fraction being $36.5 / 12=3.04 \mathrm{~Gy}$.

(ii) In a conservative situation where patient risk factors require dose reduction by $10 \%$ in order to conserve $B E D_{0.12 \%}$, all probit curves, including the human dose-response curve, have been shifted to the left by $10 \%$ at the $1 \%$ level of risk of myelopathy (as illustrated in Figure $4 \mathrm{~b})$. In this conservative situation $B E D_{0.12 \%}$ is now $89.1 \mathrm{~Gy}_{2}$, with $B E D_{\text {init }} 80 \mathrm{~Gy}$ and, thus, $B E D_{1}$ is now $89.8 \%$. Then, using the GUI which accompanies this manuscript, the resulting $B E D_{2}$ is $91.6 \%$ and the $B E D_{\text {ret }}$ for this conservative situation is $81.6 \mathrm{~Gy}_{2}$ (see Figure $5 \mathrm{~b})$. For 12 fractions, the total retreatment dose $D$ is given via

$$
81.6=D\left(1+\frac{\frac{D}{12}}{2}\right)
$$

and hence $D=33.9 \mathrm{~Gy}$.

With any additional degree of conservatism e.g. an ultra-conservative, it is likely that a higher risk of myelopathy would need to be accepted on retreatment because the initial dose of 40 Gy in 20 fractions of 2 Gy will be the usually accepted tolerance dose for an ultraconservative case. 
(iii) With individual dose fractions of $2 \mathrm{~Gy}$, and $\alpha / \beta=2 \mathrm{~Gy}$ for human spinal cord the $1 \%$ incidence of myelopathy is now associated with a dose of $54.8 \mathrm{~Gy}, B E D_{1 \%}$ and the initial biologically effective dose $B E D_{\text {init }}$ are given by:

$$
B E D_{1 \%}=54.8\left(1+\frac{2}{2}\right)=109.6 G y_{2} \text { and } B E D_{\text {init }}=40\left(1+\frac{2}{2}\right)=80 G y_{2} .
$$

Thus, the percentage initial biologically effective dose, $B E D_{1}$ is given by the equation

$$
B E D_{1}=100 \times B E D_{\text {init }} / B E D_{1 \%}=73.0 \%
$$

Given the curve fitting of the model and its parameter estimates the corresponding value for the percentage retreatment biologically effective dose one can read off, $B E D_{2}(2)$ from Figure 5c. Using a non-conservative approach, the line in Figure 5c, generated from the intersection of $B E D_{1}=73.0 \%$ with the non-conservative 2-year model prediction generates a predicted $B E D_{2}(2)$ value of $92.8 \%$ (see Figure $5 \mathrm{c}$ ). Since the nominal $1 \%$ incidence level biologically effective dose $B E D_{1 \%}$ is $109.6 \mathrm{~Gy}_{2}$ the associated retreatment biologically effective dose is $101.7 \mathrm{~Gy}_{2}$.

Hence the retreatment biologically effective dose $\left(B E D_{\text {ret }}\right)$ should be $92.8 \%$ of $109.6 \mathrm{~Gy}_{2}$; namely $101.7 \mathrm{~Gy}_{2}$ delivered using a suitable dose-fractionation schedule to be derived using this $B E D_{\text {ret }}$ value. Alternatively, in order to retain consistent 2 Gy fractions would again involve rounding up or down due to fractionation discreteness. Rounding up would imply a $B E D_{\text {ret }}$ of $104 \mathrm{~Gy}_{2}$, a dose of 52 Gy delivered in 26 fractions of $2 \mathrm{~Gy}$. However, rounding down would mean using a $B E D_{\text {ret }}$ of $100 \mathrm{~Gy}_{2}$ or a dose of $50 \mathrm{~Gy}$ in 25 fractions of $2 \mathrm{~Gy}$. It should be recognised that the small increase in dose produced by rounding up in this case would be associated with a very small increase in the level of predicted risk accepted $(p=$ $0.01562)$, while rounding down would reduce the predicted risk ( $p=0.00717)$, from what was seen as the acceptable risk of $1 \%(p=0.01)$. Again, in a palliative situation further 
reductions in the number of dose fractions might be appropriate, which for a course of 12 equal fractions for the total retreatment dose $D$ would be

$$
101.7=D\left(1+\frac{\frac{D}{12}}{2}\right)
$$

The solution for $D$ is $38.8 \mathrm{~Gy}$, the dose per fraction being $38.8 / 12=3.2 \mathrm{~Gy}$.

Worked example 2: A patient treated for an intra-thoracic malignancy received a dose of 53 Gy to the spinal cord given in 35 fractions, close to conventionally accepted spinal cord 'tolerance'. Due to recurrence, with a very significant risk of tumour spinal cord compression, further radiotherapy is needed 2 years later. Determine the safe retreatment dose for the spinal cord $(\alpha / \beta=2 \mathrm{~Gy})$ with:

(i) the use of the non-conservative model with a $1 \%$ myelopathy risk being considered clinically acceptable at retreatment, which corresponds to a BED of $109.6 \mathrm{~Gy}_{2}$;

(ii) again, using the non-conservative model but on this occasion only wanting to accept a $0.5 \%$ risk of the development of myelopathy following retreatment (revised BED for a lower level of acceptable risk of $0.5 \%-106.2 \mathrm{~Gy}_{2}$; see table 3 ). In each case the acceptable retreatment dose to the spinal cord should be given in 10, 20 or 30 fractions.

\section{Solution}

(i) From the GUI, and myelopathy risk associated with $p=0.01, B E D_{1}=85.0 \%, B E D_{2}=$ $89.7 \%$ and, thus, $B E D_{\text {ret }}=98.3 \mathrm{~Gy}_{2}$, which could be delivered as total retreatment doses of 35.5 Gy in 10 fractions of 3.5 Gy, 45.8 Gy in 20 fractions of $2.3 \mathrm{~Gy}$ and 52.4 Gy in 30 fractions of $1.8 \mathrm{~Gy}$ would be required, with dose/fraction expressed to 2 significant figures. 
(ii) For $p=0.005, B E D_{1}=87.7 \%, B E D_{2}=89.0 \%$ and, thus, $B E D_{\text {ret }}=94.5 \mathrm{~Gy}_{2}$. Hence total retreatment doses of $34.6 \mathrm{~Gy}$ in 10 fractions of $3.5 \mathrm{~Gy}, 44.7 \mathrm{~Gy}$ in 20 fractions of $2.2 \mathrm{~Gy}$ and 51.1 Gy in 30 fractions of 1.7 Gy would be required, once more with dose/fraction expressed to 2 significant figures.

\subsection{Model Sensitivity}

As tabulated in Table 2, consideration is given to the sensitivity of the predictions for $B E D_{2}$ to (i) example changes in the input factors $\overline{B E D}, s_{0}$ or $s_{1}$ (ii) a $10 \%$ shift in the dose-effect curves that results from conservatism or (iii) changing the nominal selection of a $1 \%$ incidence of myelopathy being acceptable on retreatment to a higher level of $10 \%$. Critically, in all cases $B E D_{2}$ did not change significantly.

Since the monkey data is being used in the same way as the human data, and by using the human probability of incidence with the monkey data, the change in the $B E D_{2}$ vs $B E D_{1}$ curve is so small as to be almost within the rounding process for 2 significant figure accuracy. Hence all predictions are essentially based on this relationship, which ultimately is dictated by (i) the probit curve from Ang et al (2001) original data and (ii) the monkey recovery function $r(t)$, which is remarkably robust to changes in the value of the parameters and conservatism that are within clinical relevance. This robustness increases confidence in the model.

\section{Discussion}

The proposed model allows an estimation of the recovery of the spinal cord after an initial radiation exposure, over time periods ranging from 70 days up to 3 years after the initial radiation exposure. The model is also in a form in which the experimental data can be fitted empirically, without reliance on any precise biological mechanisms that are at present 
incompletely understood. Although further experiments have already been proposed to study the mechanisms (Jones and Hopewell, 2014) it seems unlikely that these will be performed unless major changes in the emphasis and funding of research take place. To provide further advances without more basic animal based research, the collection of more data on outcomes in human is essential and the present model provides a good template on which to base any new clinical data sets. Until this is available, clinicians will need to rely on very cautious estimates of doses associated with a given risk of myelopathy, as set out in the present study.

The model exhibits no observed extremes or ill-understood sensitivity behaviours that would militate against its use. Furthermore, it also suggests how very late recovery effects could develop when the size of the initial BED delivered is below some threshold. In any clinical situation this approach must be used cautiously, particularly if an individual patient's response could be changed due to other risk factors, for example by extremes of age, significant previous chemotherapy or intervening surgical procedures. Clinicians may then need to introduce further reductions in re-treatment dose schedules, as well as choose the best treatment modality for the proposed BED to be delivered. Conservative fitting of some of the functions in the model, with under-estimations on the graphical plots have been given as suggestions, but these can be further over-ridden on medical advice by either reducing the estimated total doses and/or reducing the dose per fraction.

The cautious approach adopted was based on allowing for additional considerations, such as potential outliers in the studies; however, there may be reasons why the incidence of myelopathy could be higher in humans than in primates: these include age, diet and lifestyle factors. Consequently, reductions in the predicted dose by a further arbitrary $10 \%$ or $20 \%$ might be considered in more critical clinical circumstances, especially at extremes of age and where tissue tolerance might be expected to differ. In this situation adjustment of the actual tolerance dose should also be considered. 
Clinical decisions will be required if this form of model is used not only for re-treatment of other parts of the CNS, some of which have similar tolerance to spinal cord (e.g. brain stem and optic chiasm), but also for cortical brain regions where the tolerance is normally accepted to be higher at 60 Gy given in 30 fractions of $2 \mathrm{~Gy}$.

In addition, in recent years, re-treatment of the CNS has increasingly been delivered using radiosurgical techniques (RS) on the basis that this approach results in a reduction in the volume of previously uniformly exposed tissue to the peak treatment dose. The only experimental study to address this issue has been carried out using a spinal cord model in pigs of the Yucatan mini-pig strain (Medin et al., 2012). Animals were initially irradiated to a uniform dose of $30 \mathrm{~Gy}$ given in 10 fractions of $3 \mathrm{~Gy}$; they were partially re-irradiated, 1 year later, with graded single treatments, using a range of radiosurgical techniques. Animals receiving the single radiosurgical treatments acted as controls in this study. The conclusion was that the initial uniform dose was essentially recovered after 1 year, although a relatively small number of animals were used at each dose point. The initial uniform fractionated dose of $30 \mathrm{~Gy}$ is commonly delivered to humans (BED $75 \mathrm{~Gy}_{2}$ ), representing $75 \%$ of spinal cord tolerance in humans $\left[B E D_{1}=75 \%\right]$. However, it seems likely that spinal cord tolerance in the pig, like that of the Resus Monkey, may be higher than that of humans. In mature pigs of the Large English White strain (Millar et al., 2015), irradiated uniformly in a single treatment session, myelopathy did not occur after 19 Gy exposures, delivered in approximately 26 min; the $E D_{\mathbf{5 0}}$ was $21.13 \mathrm{~Gy}$. While it is not really possible to make a direct comparison between the two pig studies, de novo RS irradiation with an average peak dose of 19 Gy was given in a shorter time of 9-13 min (Medin, personal communication, 2012). This resulted in 1 of 5 animals developing myelopathy (20\%). Treatment times are known to be of particular importance in single treatment exposures (Millar et al., 2015) and repair data from rodents has been found to be applicable. Doses of less than 18.8 Gy were not associated with any 
cases of myelopathy in the Yucatan pig retreatment study. Based on the assumption that a single treatment of 18 Gy represents 'tolerance' in the pig spinal cord then the initial fractionated dose of $30 \mathrm{~Gy}$ 'only' represents $\sim 40 \%$ of 'tolerance' in this species [BED $\sim 40$ $(\%)]$. Based on the predictions of the presently proposed model this would imply that after 1 year the retreatment dose would be greater than $90 \%$ of 'tolerance' in the pig but this would not be the case in humans where the maximum suggested retreatment dose would only be $80 \%$ of human spinal cord tolerance. It would take humans approximately 2 years to achieve the same level of recovery (>90\%), simply because 30 Gy in 10 fractions represents a greater percentage of the reference tolerance dose in humans (see Figure 3), pointing to the importance of using rations of BED values for the determination of $B E D_{2}$ and ultimately $B E D_{\text {ret }}$ (see equation (1b)). The difference of less than $10 \%$ at 1 year would be undetectable using the study design adopted for the pig study. However, it is of interest to note that in the retreatment group, a SR dose of 19 Gy was associated with a higher incidence of myelopathy in 2 of 5 animals (40\%) and that these were delivered in a wider range of treatment times, 1026 min, due to the range of different SR methods used for that particular series of animals in the study (Medin, personal communication 2012). The above discussion suggests the need for caution when interpreting results if a typical standard treatment used in humans is applied in an animal study and reninforces the importance of relative and not absolute BED values in the evaluation of retreatment dose.

Thus in summary, the impact of the rhesus monkey dataset on radiotherapy retreatment has been further considered. In particular, curve fitting techniques have ensured modeling predictions fit this data set and a probit model has been used to extrapolate between the levels of incidence in the experiments and in those more commonly accepted in the clinic; furthermore, the impact of slow recovery dynamics at low doses has also been incorporated, together with a qualitative comparison with the available rodent data. The resulting modeling 
framework is thus an improvement on the fitting procedures previously developed by Jones and Hopewell (2014), while the disadvantage of additional complexity in calculating predicted retreatment doses has been ameliorated by the supply of a GUI, which provides a retreatment dose that would be consistent with the rhesus monkey study of Ang et al (2001). Nonetheless, due conservatism and medical judgment are required for interpreting rhesus monkey data and converting the resulting predictions into a clinical setting.

\section{Acknowledgements}

The authors remember the late Kian Ang whose unique study in the rhesus monkey provided the database for the present study. In addition, one of us (JWH) gratefully acknowledges personal discussions with him, which contributed to some of the thinking in the present manuscript. TEW would like to thank St John's College, Oxford and BBSRC grant BKNXBKOO BK00.16 for their financial support. JBB and GFC gratefully acknowledge the Mathematical Institute (University of Oxford) for its hospitality and the José Castillejo Fellowship Program numbers CAS14/00383 and CAS14/00363, respectively, funded by the Spanish Ministry of Education, Culture and Sports, for their research stay at the Mathematical Institute. They also thank funding from projects supported by Ministerio de Economía y Competitividad/FEDER, Spain [grant numbers: MTM2012-31073 and MTM2015-71200-R], Consejería de Educación, Cultura y Deporte from Junta de Comunidades de Castilla-La Mancha, Spain [grant number PEII-2014-031-P] and the James

S. Mc. Donnell Foundation 21st Century Science Initiative in Mathematical and Complex Systems Approaches for Brain Cancer, USA [Collaborative-Planning Grant 220020420 and Collaborative Award 220220450].

\section{Declaration of interest}

The authors report no conflict of interest. The authors alone are responsible for the content and writing on the manuscript. 


\section{Data Archiving Statement}

In compliance with Research Council UK's open access initiative, the data in this paper is available from http://dx.doi.org/xxx/xxx

\section{Profiles}

Dr Thomas E. Woolley studied mathematics at the University of Oxford between 2004-2017 and is now a Lecturer of Applied Mathematics at Cardiff University. His research focuses on applying mathematical techniques to biological problems in pattern formation, stem cell movement, oncology and neurobiology.

Dr Juan Belmonte-Beitia. Graduate in Mathematics and Physics at Universidad Complutense de Madrid. PhD in Mathematics (2008) at University of Castilla-La Mancha. His research interests are dynamical systems, differential equations and partial differential equations and their applications to Mathematical Biology, specifically the mathematical modeling of tumor growth.

Dr Gabriel F. Calvo obtained PhD in Physics at Autonoma University, Madrid. Research activities have spanned from Nonlinear Optics to Quantum Information Processing with Photons, Nanoparticles for Biomedical Applications and Mathematical Oncology. Currently Associate Professor, Department of Mathematics and senior member at the MôLAB-Mathematical Oncology Laboratory (UCLM-University, Castilla-La Mancha).

Prof. John W. Hopewell was the Director of Radiobiological Research, University of Oxford, between 1980 and 2001. His primary research interest has been the study of different aspects of normal tissue toxicity related to radiotherapy: pathogenesis, fractionation and dose-rate, volume effects and retreatment.

Prof. Eamonn A. Gaffney is an Associate Professor, at the Mathematical Institute, University of Oxford, with interests in mathematical and computational modelling for the life sciences.

Prof Bleddyn Jones is a medically qualified radiobiologist and Professor of Clinical Radiobiology at The University of Oxford. He has made many contributions to explicit mathematical models of radiotherapy and chemotherapy, as well as their application in the clinic. He started re-treating brain and spinal tumour patients in 1991. 


\section{References}

Ang KK, Jiang GL, Feng Y, Stephens LC, Tucker SL, Price RE. 2001. Extent and kinetics of recovery of occult spinal cord injury. Int J Radiat Oncol Biol Phys 50:10131020.

Brahme A. 1984. Dosimetric precision requirements in radiation therapy. Acta Radiol Oncol 23:379-391.

Burden R, Faires JD. 2011. Numerical Analysis. Ninth Edition. Brooks/Cole, Cengage Learning, Boston.

Hopewell JW, Morris AD and Dixon-Brown A. 1987. The influence of field size on the late tolerance of the rat spinal cord to single doses of $\mathrm{x}$ rays. Brit J Radiol 60: 1099-1108.

Hornsey S, Myers R, Coultas PG, Rogers MA, White A (1981) Turnover of proliferative cells in the spinal cord after X-irradiation and its relation to time-dependent repair of radiation damage. Br J Radiol 54:1081-5

Jones B, Dale RG, Deehan C, Hopkins KI, Morgan DAL. 2001. The role of biologically effective dose (BED) in Clinical Oncology. Clinical Oncology (Royal College of Radiologists) 13:71-81.

Jones B, Grant W. 2014. Retreatment of central nervous system tumours, Clinic Onc 26:407-418.

Jones B, Hopewell JW. 2014. Alternative models for estimating the radiotherapy retreatment dose for the spinal cord, Int J Radiat Biol 90:731-741.

Kirkpatrick JP, van der Kogel AJ, Schultheiss TE. 2010. Radiation dose-volume effects in the spinal cord. Int J Radiat Oncol Biol Phys 76:S42-S49.

Lyubimova N and Hopewell JW. 2004. Experimental evidence in support of the hypothesis that damage to the vascular endothelium plays a primary role in the development of late radiation-induced CNS injury. Brit J Radiol 77:488 - 492.

Medin PM, Foster RD, van der Kogel AJ, Sayre JW, McBride WH, Solberg TD. 2012. Spinal cord tolerance to reirradiation with single-fraction radiosurgery: A swine model. Int J Radiat Oncol Biol Phys 83:1031-1037.

Millar WT, Hopewell JW, Paddick I, Lindquist C, Nordströn H, Lidberg P and Gårding J. 2015. The role of the concept of biologically effective dose (BED) in treatment planning in radiosurgery. Physica Medica: Eur J Med Phys 31:627-633.

Morris GM, Coderre JA, Hopewell JW, Micca PL, Wielpolski L. 1998. Boron neutroncapture therapy: Re-irradiation response of the rat spinal cord. Radiother Oncol 48:313317.

Morris GM and Hopewell JW. 1988. Changes in the cell kinetics of the pig epidermis after single doses of $\mathrm{x}$ rays. Brit J Radiol 61:205-211. 
Nieder C, Grosu AL, Andratschke NH, Molls M. 2005. Proposal of human spinal cord reirradiation dose based on collection of data from 40 patients. Int J Radiat Oncol Biol Phys 61:851-855.

Nieder C, Grosu AL, Andratschke NH, Molls M. 2006. Update of human spinal cord reirradiation tolerance based on additional data from 38 patients. Int $\mathrm{J}$ Radiat Oncol Biol Phys 66:1446-1449.

Sahgal A, Weinberg V, Ma L, Chang E, Chao S, Muacevic A, Gorgulho A, Soltys S, Gerszten PC, Ryu S, Angelov L, Gibbs I, Wong CS, Larson DA. 2013. Probabilities of radiation myelopathy specific to stereotactic body radiation therapy to guide safe practice. Int J Radiat Oncol Biol Phys 85:341-347.

Schultheiss TE. 2008. The radiation dose-response of the human spinal cord. Int J Radiat Oncol Biol Phys 71:1455-1459.

Trott KR, Doerr W, Facoetti A, Hopewell J, Langendijk J, Luijk PV, Ottolenghi A, Smyth V. 2012. Biological mechanisms of normal tissue damage: Importance for the design of NTCP models. Radiotherapy and Oncology 105:79-85.

van der Kogel AJ. 1989. Continuous, hyperfractionated, accelerated radiotherapy (CHART) (by Dische and Saunders): Editorial Comment. Radiother Oncol, 16: 75-77.

van der Kogel AJ, Sissingh HA, Zoetelief J. 1982. Effect of X rays and neutrons on repair and regeneration in the rat spinal cord. Int J Radiat Oncol Biol Phys 8:2095-2097.

Wong CS, Minkin S, Hill RP. 1993. Re-irradiation tolerance of rat spinal cord to fractionated X-ray doses. Radiotherapy and Oncology 28:197-202.

Wong CS, Hao Y. 1997. Long-term recovery kinetics of radiation damage in rat spinal cord. Int J Radiat Oncol Biol Phys 37:171-179.

Zeman W, Carsten A, Biondo S (1964) Cytochemistry of delayed radionecrosis of the murine spinal cord. In Response of the Nervous System to Ionizing Radiation (eds Haley TJ, Snider RS), p 105. Academic Press, New York. 
Table 1: Summary of definitions, of dose related parameters (A) and the assumed recovery (B) variables, used in analysis.
Symbol
Definition
Units
Comment

\begin{tabular}{|c|c|c|c|c|}
\hline (A) & $\mathrm{D}_{\text {init }}$ & $\begin{array}{l}\text { Total dose used in initial } \\
\text { treatment }\end{array}$ & Gy & $\begin{array}{l}\text { In } n \text { fractions of dose } \\
d\end{array}$ \\
\hline & $\mathrm{D}_{\text {ret }}$ & $\begin{array}{l}\text { Total dose used in re- } \\
\text { treatment }\end{array}$ & Gy & $\begin{array}{l}\text { In } \boldsymbol{n} \text { fractions of dose } \\
d \text { (may be different for } \\
\text { the re-treatment } \\
\text { compared with initial } \\
\text { irradiation). }\end{array}$ \\
\hline & $\mathrm{BED}_{\text {init }}$ & $\begin{array}{l}\text { The BED of the initial } \\
\text { treatment }\end{array}$ & $\mathrm{Gy}_{2}$ & $\alpha / \beta$ of 2 Gy used \\
\hline & $\mathrm{BED}_{\text {ret }}$ & $\begin{array}{l}\text { The BED of the re- } \\
\text { treatment }\end{array}$ & $\mathrm{Gy}_{2}$ & $\alpha / \beta$ of 2 Gy used \\
\hline & $\mathrm{BED}_{\mathrm{R} \%}$ & $\begin{array}{l}\text { The BED that should result } \\
\text { in a risk of } \mathrm{R} \%\end{array}$ & $\mathrm{~Gy}_{2}$ & $\alpha / \beta$ of 2 Gy used \\
\hline & $\mathrm{BED}_{1}(\%)$ & $\begin{array}{l}\text { The ratio of } \mathrm{BED}_{\text {init }} \text { to } \\
\mathrm{BED}_{\mathrm{R} \%} \text { expressed as a } \\
\text { percentage }\end{array}$ & dimensionless & $\begin{array}{l}\text { to provide } \mathrm{BED}_{2}(\%) \\
\text { value }\end{array}$ \\
\hline
\end{tabular}

\begin{tabular}{|c|c|c|c|c|}
\hline & $\mathrm{BED}_{2}(\%)$ & $\begin{array}{l}\text { The ratio of } \mathrm{BED}_{\text {ret }} \text { to } \\
\mathrm{BED}_{\mathrm{R}} \% \text { expressed as a } \\
\text { percentage }\end{array}$ & dimensionless & $\begin{array}{l}\mathrm{BED}_{2}(\%) \text { needs to be } \\
\text { converted into BED ret } \\
\text { and then } \mathrm{D}_{\text {ret }} \text { for a } \\
\text { selected value of } n \\
\text { and/or } d \text {. }\end{array}$ \\
\hline (B) & $\overline{B E D}$ & $\begin{array}{l}\text { Transition point between } \\
\text { delayed and normal } \\
\text { recovery. }\end{array}$ & $\begin{array}{l}\text { Function of } \\
\operatorname{BED}_{1}(\%)\end{array}$ & $\begin{array}{l}\text { conservative value of } \\
35 \% \text { used } *\end{array}$ \\
\hline & $\mathrm{t}_{\mathrm{IRO}}$ & $\begin{array}{l}\text { Time to initial onset of } \\
\text { recovery }\end{array}$ & Years & $\begin{array}{l}\text { Value of } 0.19 \text { years } \\
\text { adopted }\end{array}$ \\
\hline & $\mathrm{r}(\mathrm{t})$ & $\begin{array}{l}\text { recovery function that } \\
\text { depends on the elapsed } \\
\text { time, }\end{array}$ & dimensionless & ' $t$ ' values years \\
\hline & $s_{0}$ & Transition steepness & dimensionless & Value of 0.15 used * \\
\hline & $s_{1}$ & $\begin{array}{l}\text { Modulator scale for low } \\
\text { BED }_{1} \text { recovery. }\end{array}$ & dimensionless & Value of used $0.1^{*}$ \\
\hline
\end{tabular}

*the tablulated parameter values should only be taken as suggestions, they are indicative of the correct size expectation expected in clinical practice and valiation in their values dose not have a major inpact on the predictions for $\mathrm{BED}_{2}$ 
Table 2: Using the top row as the set of base parameters each further line changes one parameter to illustrate local parameter insensitivity with $B E D_{2}$ and $B E D_{\text {ret }}$ evaluated at $t=2$ years. It can be seen that there is only a small sensitivity to changes in $\overline{B E D}, s_{0}$ and $s_{1}$, as expected. $\dagger$ is used to specify which variable has changed on each line (when compared with the top row) whilst $*$ denotes a non-conservative treatment and $* *$ denotes a conservative treatment, i.e. a $10 \%$ shift in the dose-effect curves at a $1 \%$ incidence of risk of myelopathy. Note that the Monkey $B E D_{R \%}$ is determined using the rhesus monkey dose per fraction of $d=2.2$ Gy (Ang et al. 2001) rather than the standard human clinical dose fraction of $d=$ 2 Gy.

\begin{tabular}{|c|c|c|c|c|c|c|c|c|c|}
\hline $\begin{array}{c}\text { Human } \\
B E D_{R \%} \\
\left(G_{2}\right)\end{array}$ & $\begin{array}{c}\text { Monkey } \\
B E D_{R} \% \\
\left(G_{2}\right) \\
\end{array}$ & $p$ & $R$ & $s_{0}$ & $s_{1}$ & $\overline{B E D}$ & $\begin{array}{c}B E D_{\text {init }} \\
\quad\left(\mathbf{G y}_{2}\right)\end{array}$ & $\begin{array}{c}B E D_{2} \\
(\%)\end{array}$ & $\begin{array}{c}B E D_{\text {ret }} \\
\left(\mathbf{G y}_{2}\right)\end{array}$ \\
\hline $109.60 *$ & 127.9 & 0.01 & 1 & 0.15 & 0.1 & 35 & 80 & 92.8 & 101.7 \\
\hline $109.60 *$ & 127.9 & 0.01 & 1 & 0.15 & 0.1 & $15 \dagger$ & 80 & 92.8 & 101.7 \\
\hline $109.60 *$ & 127.9 & 0.01 & 1 & 0.15 & $0.25 \dagger$ & 35 & 80 & 92.8 & 101.7 \\
\hline $109.60 *$ & 127.9 & 0.01 & 1 & $0.25 \dagger$ & 0.1 & 35 & 80 & 92.8 & 101.7 \\
\hline $98.64 * *$ & 115.1 & 0.01 & 1 & 0.15 & 0.1 & 35 & 80 & 92.7 & 91.5 \\
\hline $123.66^{*}$ & 142.7 & $0.1 \dagger$ & $10 \dagger$ & 0.15 & 0.1 & 35 & 80 & 93.7 & 115.8 \\
\hline $\begin{array}{c}112.70 * \\
*\end{array}$ & 129.9 & $0.1 \dagger$ & $10 \dagger$ & 0.15 & 0.1 & 35 & 80 & 93.7 & 105.6 \\
\hline
\end{tabular}


Table 3: Variation in the total non-conservative dose associated with irradiation at 2

Gy/fraction and the estimated incidence of radiation myelopathy above the accepted tolerance doses (BED values) in humans based on the assumptions in the text with the $1 \%$ incidence of myelopathy normalised to $54.8 \mathrm{~Gy}$ in $2 \mathrm{~Gy}$ fractions. The associated BED values are calculated for the original non-conservative situation and for situations where there are other associated risk factors such that the BED delivered is reduced by $10 \%$ (conservative) or $20 \%$ (ultra-conservative) for the same risk of myelopathy based on two assumptions, either a simple 10 or $20 \%$ reduction in the total dose for the different levels of risk or a 10 or $20 \%$ left shift of the dose effect curve at a $1 \%$ incidence of risk of myelopathy. Note that in certain cases of very low risk and very high conservatism it is possible that the initial dose would exceed the BED of the specified tolerances. Such cases are extrapolating outside the model's domain of reliability and should be disregarded. Continued use of the model and its GUI then rests on first relaxing either the level of risk or conservatism.

\begin{tabular}{|c|c|c|c|c|c|c|}
\hline \multirow[t]{2}{*}{$\begin{array}{l}\text { Risk of } \\
\text { myelopathy } \\
\text { tolerance, } R \\
(\%)\end{array}$} & \multirow[t]{2}{*}{$\begin{array}{l}\text { Total dose }(2 \\
\text { Gy/fraction, } \\
\text { non- } \\
\text { conservative) }\end{array}$} & \multirow[t]{2}{*}{$\begin{array}{l}\text { For non- } \\
\text { conservative } \\
\text { policy BED } \\
\text { (Gy2) }\end{array}$} & \multicolumn{2}{|c|}{$\begin{array}{l}\text { For } \\
\text { conservative } \\
\text { policy BED } \\
\text { (Gy2) }\end{array}$} & \multicolumn{2}{|c|}{$\begin{array}{l}\text { For ultra- } \\
\text { conservative } \\
\text { policy BED } \\
(\text { Gy2) }\end{array}$} \\
\hline & & & $\begin{array}{l}-10 \% \\
\text { shift }\end{array}$ & $-10 \% *$ & $\begin{array}{l}-20 \% \\
\text { shift }\end{array}$ & $-20 \% *$ \\
\hline 'Tolerance' & 50.00 & 100.00 & 89.04 & 90.00 & 78.08 & 80.00 \\
\hline 0.25 & 51.57 & 103.13 & 92.17 & 92.82 & 81.21 & 82.5 \\
\hline 0.5 & 53.12 & 106.24 & 95.28 & 95.62 & 84.32 & 84.99 \\
\hline 1.0 & 54.80 & 109.60 & 98.64 & 98.64 & 87.68 & 87.68 \\
\hline 2.0 & 56.64 & 113.27 & 102.31 & 102.0 & 91.35 & 90.67 \\
\hline 4.0 & 58.68 & 117.35 & 106.39 & 105.62 & 95.43 & 93.88 \\
\hline 6.0 & 59.99 & 119.98 & 109.02 & 107.98 & 98.06 & 95.98 \\
\hline 8.0 & 61.00 & 122.00 & 111.04 & 109.8 & 100.08 & 97.6 \\
\hline 10.0 & 61.83 & 123.66 & 112.70 & 111.29 & 101.74 & 98.93 \\
\hline
\end{tabular}

*conservative and ultra-conservative BED values for different levels of risk of myelopathy that might be considered acceptable on retreatment. Values obtained by a 10 or $20 \%$ left shift of the non-conservative dose-effect curve, nominally drawn parallel at the $1 \%$ risk level, total dose $54.8 \mathrm{~Gy}$, are compared with those obtained simply reducing the original doses for the different non-conservative risks by 10 or $20 \%$. The approach based on the nominal approach to the shift of the dose-effect curves produces a slightly greater degree of conservatism for levels of risk $<1 \%$ ( $1.13 \%$ for 'tolerance' in the ultra-conservative situation) and slightly less conservative for risks $>1 \%$ ( $2.84 \%$ for the $10 \%$ risk ultra conservative situation) when compared with the simple 10 and $20 \%$ reduction in the proposed total doses for the different levels of risk in the non-conservative situation. 

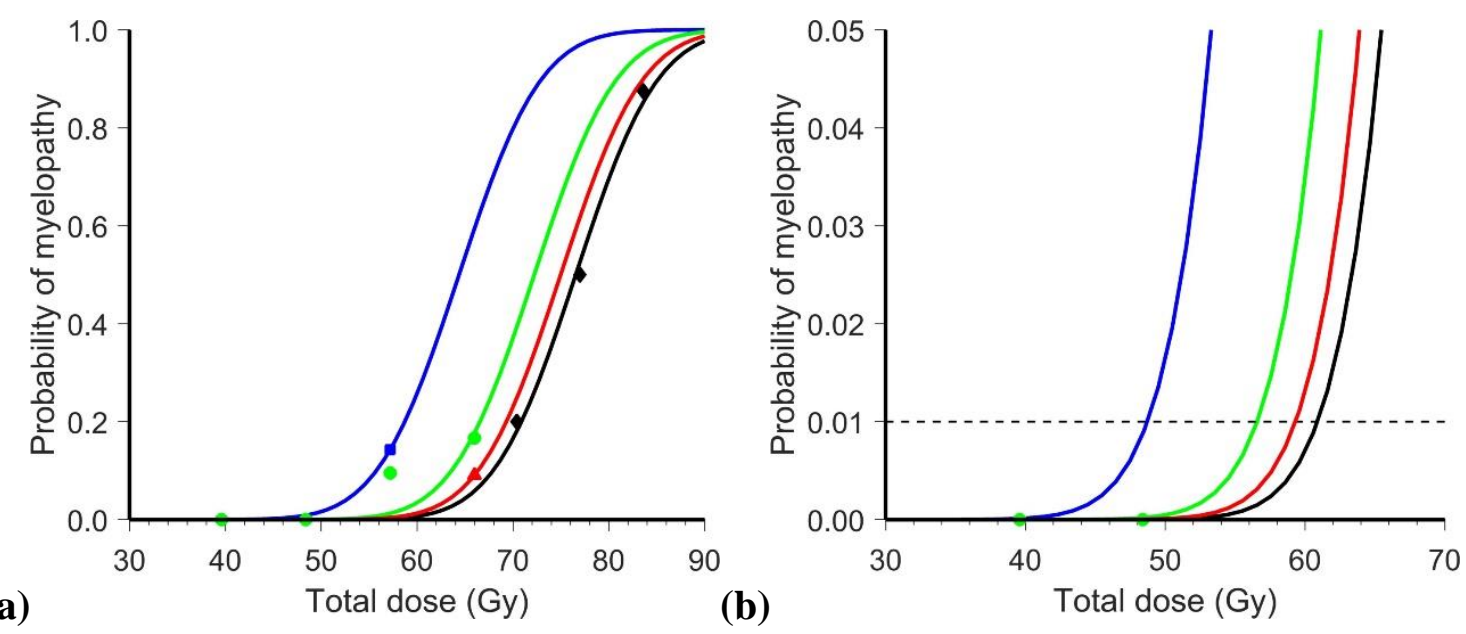

Figure 1. (a) Dose-response data for radiation myelopathy in the Rhesus Monkey (Ang et. al., 2001) following single course radiation exposure given in $2.2 \mathrm{~Gy} /$ fraction (black) and after re-irradiation at 1, 2 and 3 years following an initial dose of 44 Gy given in 20 fractions of $2.2 \mathrm{~Gy}$ (blue, green and red, respectively). The curves represent a fit using the probit doseresponse model, in which all the curves were fixed to be parallel at the $50 \%$ incidence level.

(b) Magnification of the curves in Fig 1a for low probabilities of myelopathy; the dashed line corresponds to $p=0.01$ (that is a $R=1 \%$ incidence of myelopathy). Each fitted curve is specified by two parameters, $\gamma_{50}$ and $D_{50}$, using equation (5), noting $\gamma_{50} / D_{50}$ is determined from the black, single course curve and inherited by all other curves. The fitted parameters, $\gamma_{50}$ and $D_{50}$, were, respectively, 11.4 and $76.6 \mathrm{~Gy}$ for the single course irradiation curve (black); 9.6 and $64.4 \mathrm{~Gy}$ for the 1 year re-irradiation curve (blue); 10.7 and 72.2 Gy for the 2 years re-irradiation curve (green) and 11.1 and 75.0 Gy for the 3 years re-irradiation curve (red). These curves are used to generate the values of $r(t)$ discussed in section (2.4), and subsequently, the non-conservative model. 


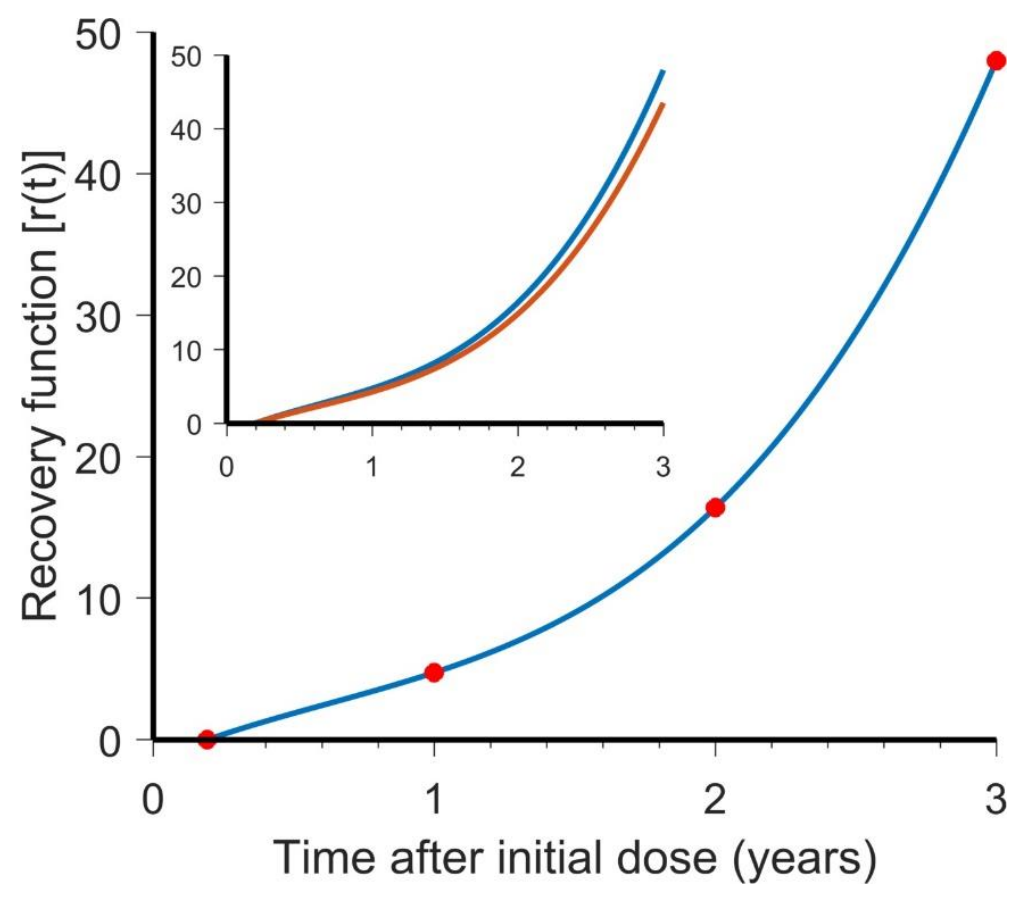

Figure 2. Illustration of the recovery function fitted to the experimental data from the Rhesus monkey of Ang et al. (2001) calculated from the probability curves shown in Figure 1. Note that the recovery function is zero up to time, $t_{I R O}=70 / 365$ years. Other parameters: $s_{0}=$ $0.15, s_{1}=0.1$ and $\overline{B E D}=35 \%$, with a probability of myelopathy set at $p=0.01$. The coordinate points and the definition of the curve are given in the text. The inset shows the recovery function with $p=0.01$ (blue) and $p=0.1$ (red), demonstrating that the recovery function is relatively insensitive to changes in the value of $p$. 

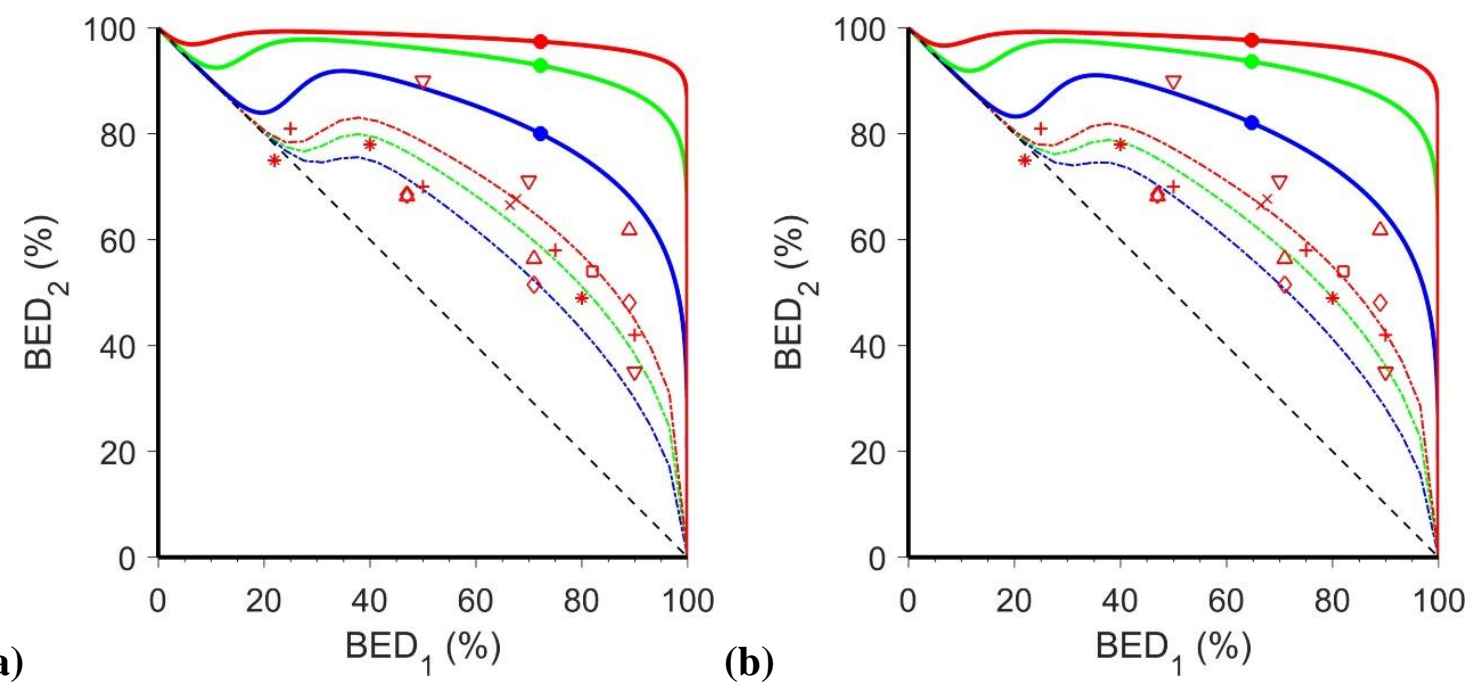

Figure 3. Predicted dependence between the initial and the re-irradiation doses expressed as a percentage of BED for the specific risk of myelopathy considered acceptable on re-irradiation (a) $p=0.01$ and (b) $p=0.1$, using the non-conservative model. The dashed diagonal line corresponds to the situation with no recovery between the initial and the re-irradiation dose. The solid lines illustrate the fitted curves for elapse times of 1 (blue), 2 (green) or 3 (red) years between the initial and re-irradiation dose. The dash-dotted lines illustrate the fitted curves for elapsed times of 4 (blue), 5 (green) or 6 (red) months. These shorter time intervals show that the curves cover the range of the rodent data points, based on $\mathrm{ED}_{50}$ values $(p=$ 0.5), which are illustrated by the red symbols: Morris et al., 1998; *, $\square$ : Wong and Hao, 1997; $\diamond, \Delta$ : van der Kogel et al. 1982; X, $\nabla$ : Wong et al. 1993; +. The solid circle symbols are data points for the rhesus monkey derived from calculations outlined in the text. 

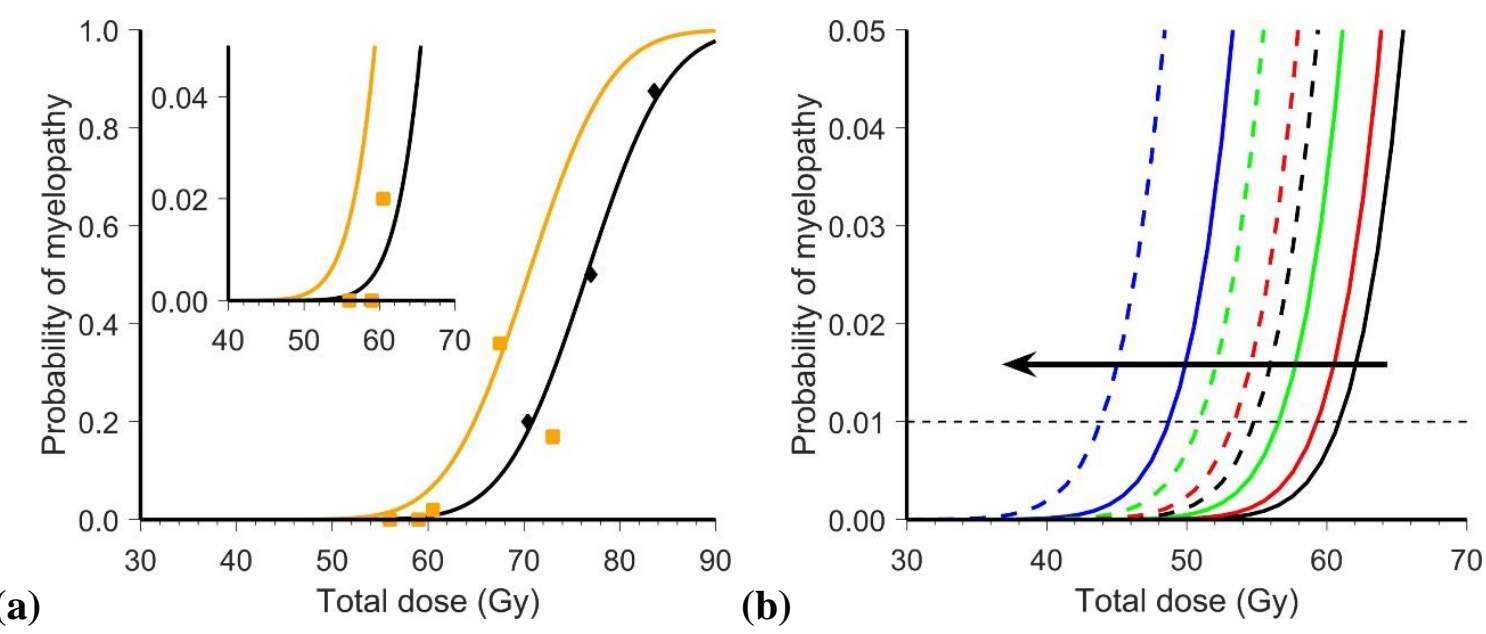

Figure 4. (a) Data for the incidence of radiation myelopathy (orange squares) in humans (Ang, pers. comm. 1996) compared with those for the rhesus monkey (black diamonds/black line) as previously illustrated in Figure 1 . The solid orange line is the probit curve fit to the orange square data point, ignoring the rightmost outlier human data point and fitted parallel to the curve for the rhesus monkey, at a probability of 0.5 , thus providing a degree of conservatism for the dose/effect estimate. Based on this curve the dose associated with $p=$ 0.01 incidence of myelopathy is $54.8 \mathrm{~Gy}$. The inset zooms in on the region of the dose-effect curve where the incidence satisfies $p<0.05$. (b) For a more conservative model prediction, the dose-response curves for radiation myelopathy in the Rhesus Monkey (Ang et. al., 2001) given in Figure $1 \mathrm{~b}$ for retreatment and for an initial treatment have been shifted to the left by $10 \%$ at a risk level for myelopathy of $p=0.01$. The solid line curves represent the original data and the dashed curves represent the $10 \%$ conservative shift. 

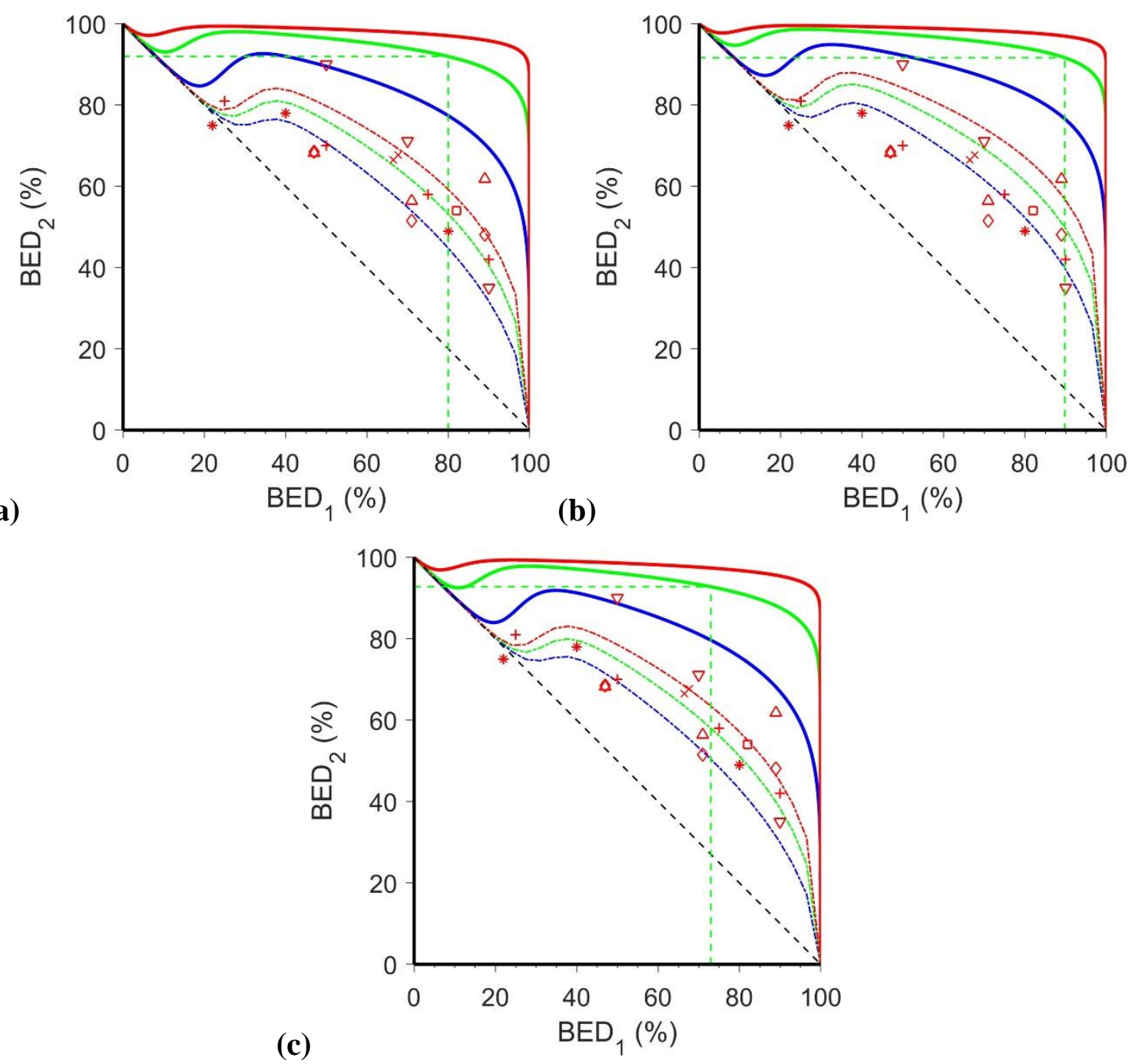

Figure 5. Plots of predicted relationships between the initial and re-irradiation percentage BED values. Figures (a) - (c) correspond to worked example 1 parts (i)-(iii), respectively. For (a), (b) and (c) the data points are the same as shown in Fig. 3, while the curves are constructed analogously. The straight thin green dashed lines correspond to the $B E D_{1}$ and $B E D_{2}$ values of the, respective, solutions. 


\section{Appendix A. Further justification for the functional form for the relation between $B E D_{2}$ and $B E D_{1}$}

In equations (3) and (4) of the materials and methods section, the function $f\left(B E D_{1}, r(t, p)\right)$ controls the transition between initial BED dependent early and late recovery, with $f\left(B E D_{1}, r(t)\right) \approx 1$ corresponding to the recovery described by Jones and Hopewell (2014), while $f\left(B E D_{1}, r(t)\right) \approx 0$ entails essentially no recovery. Thus, it is essential to consider where this function switches between zero and unity to provide further insight into the behaviour of the present model.

For the early stages of recovery, with a small initial dose, such that $B E D_{1}<\overline{B E D}$ and $r(t) \ll$ 1 then, given that the transition steepness, $s_{0}$, is sufficiently large $f\left(B E D_{1}, r(t)\right) \approx 0$, and thus

$$
B E D_{2} \approx 100\left(1-\frac{B E D_{1}}{100}\right)
$$

which corresponds to no recovery. However, since the value of $r(t)$ increases with time, over a timescale modulated by $s_{1}$, then eventually

$$
B E D_{1}>\frac{\overline{B E D}}{1+s_{1} \cdot r(t)} .
$$

Thus, again for $s_{0}$ sufficiently large $f\left(B E D_{1}, r(t)\right) \approx 1$, for long times and thus

$$
B E D_{2} \approx 100\left(1-\frac{B E D_{1}}{100}\right)^{\frac{1}{r(t)+1}}
$$

with the right hand side of the equation representing the original model of Jones and Hopewell (2014). 


\section{Appendix B. The Graphical User Interface (GUI).}

In association with this paper, a standalone GUI can be used to perform the calculations for the examples in the main text, allowing for alterations in the parameters and the level of conservatism adopted. MATLAB source files have been provided that produce the GUI, allowing code modification. The codes have been tested on Ubuntu 14.04, 16.06 and on 32bit and 64bit Windows 7 using both MATLAB versions R2013a, R2015a and R2016b.

If MATLAB is unavailable, the GUI can nonetheless be run via a simple installation, as tested on Windows 7, by downloading and run the Supplementary Information file “MyAppInstaller_mcr.exe”. This 'exe' file will download and install the appropriate files. Once installed, the application is termed "BED_Calculator.exe". For Windows computers, the default location of the executable "BED_Calculator.exe" file can be found in

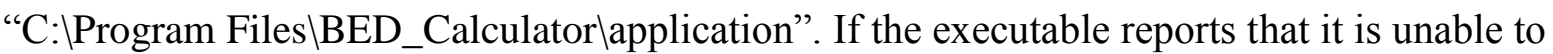
find ".dll" files, the System Path needs to be adjusted. One way of do this is to bring up "Advanced system settings" in "System", which can be found in the control panel. This produces a menu with a number of tabs, one being "Advanced". At the bottom of this tab click "Environmental Variables". This should bring up a window at the bottom of which there should be a pane with the title "System variables". Scroll within this pane to find the variable called "Path". Highlight "Path" and press "Edit". Another box should appear and in the editable box called "Variable value" add the location of the MATLAB runtime files that have just been downloaded. If the default location has not been changed then simply add the line “;C:IProgram Files\MATLAB\MATLAB Runtimelv90\runtimelwin64” or ";C:IProgram Files\MATLAB\MATLAB Runtimelv90|runtimelwin32” to the end of the value, depending on whether the 32 or 64 bit version has been installed. Finally, click "OK" on all the boxes to confirm the changes. 


\section{B.1 Using the GUI}

On the left handleft-hand panel of the GUI, the predictions are given for specific input data. Namely, it provides a graphical illustration of the $B E D_{1}$ and $B E D_{2}$ curve together with four fields: the $B E D_{\text {init }}$ and $B E D_{\text {ret }}$ values in units of $\mathrm{Gy}_{2}$ and the $B E D_{1}$ and $B E D_{2}$ values which are given as percentages of the BED associated with an acceptable level of incidence of myelopathy on retreatment, which for the purposes of the majority of the calculations in this manuscript has nominally been set at a $1 \%$ risk, $B E D_{1 \%}$. However, this acceptable level of risk, and the associated $\left(B E D_{R \%}\right)$ can be changed with minimal effect on the recovery function.

In the centre panel of the GUI there are boxes that can be manually changed to suit the user's input. Each input box is beside its definition. The default values match the values in worked example 1(iii), Section 3.3. There is also a button at the top of the central panel which 'toggles' the visibility of the rodent data, as described in the caption of Fig. 3.

It should be noted that the user can only specify one of the "Risk of myelopathy" and "Nominal tolerated $B E D_{R \%}$ " boxes as an input. The input box is selected by pressing the appropriate "Use in calculation" radio button. Once the user has specified one of these values, the other is calculated as part of the GUI output, once the "Calculate" button is pressed.

The sliders on the right panel of the GUI represent variables which can be altered continuously by altering the slider positions along the bar. The numerical value to the left of each bar gives its current value.

Finally, at the bottom of the GUI, there is the "Calculate" button, which will update the graph and output values on the left panel with the resulting values calculated using the numbers currently appearing in the boxes and the sliders. 
If the "Show rodent data" button and "Calculate" button are pressed with the default parameter set on the GUI then the image in Fig. B2 will be generated. This is the solution to Worked Example 1(iii), presented in Section 3.3.1. Along with the rodent data and the 1, 2 and 3 year predictions for $B E D_{2}$ are plotted. Further, more a black trace mark pinpoints the input $B E D_{1}$ and the output $B E D_{2}$. The retreatment dose per fraction is also determined and printed in the lower left-hand region of the screen. If the user increases the conservative factor slider to $10 \%$, they will produce the conservative estimate as mentioned in the main text example.

Given familiarity with the basics of the GUI, the user can manipulate the inputs for their own calculations. In particular, in Figure B3 we see the influence of changing the initial dose to 44 Gy, the number of fractions to 22 , the variables to $s_{0}=1, s_{1}=0.01, \overline{B E D}=50$, reducing the time until retreatment to 0.8 year and increasing the conservatism to $10 \%$. Comparing Figs $\mathrm{B} 2$ and $\mathrm{B} 3$ illustrates that these inputs cause $B E D_{1}$ to be larger and the corresponding output $B E D_{2}$ to be smaller, due to the monotonic decreasing shape of the curve after $B E D_{1}=50 \%$.

\section{Appendix C.}

\section{Coefficients of the recovery function.}

Equations for calculating the coefficients of the recovery function $r(t)$, based on delayed recovery time, $t_{I R O}$, and the derived values $r(1), r(2)$ and $r(3)$ are

$$
\begin{gathered}
a=3 \frac{t_{I R O} r(1)}{t_{I R O}-1}-3 \frac{t_{I R O} r(2)}{t_{I R O}-2}+\frac{r(3) t_{I R O}}{t_{I R O}-3} \\
b=-1 / 2 \frac{\left(5 t_{I R O}+6\right) r(1)}{t_{I R O}-1}+\frac{\left(4 t_{I R O}+3\right) r(2)}{t_{I R O}-2}-1 / 2 \frac{\left(3 t_{I R O}+2\right) r(3)}{t_{I R O}-3}, \\
c=1 / 2 \frac{\left(t_{I R O}+5\right) r(1)}{t_{I R O}-1}-\frac{\left(t_{I R O}+4\right) r(2)}{t_{I R O}-2}+1 / 2 \frac{\left(t_{I R O}+3\right) r(3)}{t_{I R O}-3}, \\
d=-1 / 2 \frac{r(1)}{t_{I R O}-1}+\frac{r(2)}{t_{I R O}-2}-1 / 2 \frac{r(3)}{t_{I R O}-3} .
\end{gathered}
$$




\section{Appendix D.}

When applying a conservative factor, all the dose-response curves have been shifted to the left by the chosen conservative factor, such that all curves remain parallel. For illustrative purposes in the main manuscript, the conservative reduction was calculated at the level of $1 \%$ risk of incidence of myelopathy. Specifically, a $1 \%$ incidence corresponds to a 54.8Gy total dose, given in 2 Gy fractions, in humans (see Figure 4 and Section 3.2). A conservative reduction of $C \%$ then specifies that a total dose of $54.8(1-C / 100)$ Gy would correspond to that $1 \%$ risk (see Section 3.2). Hence, the degree of the conservative shift depends on the specified level of risk selected (1\% was taken for illustrative purposes in the main manuscript). Here, it is shown that altering the level of risk at which the shift is applied has very little influence of the resulting BED values.

The impact of reducing the human dose response curve (orange curve: same as the orange curve in Figure 4) by 10\% and 20\%, nominal conservative and ultra-conservatism, respectively, for a myelopathy risk of $1 \%, 5 \%, 10 \%$ and $50 \%$ is illustrated in Figure D. Note that a $50 \%$ risk is above what would ever be considered a clinically acceptable level of risk, however, this is the level of risk was associated with the rodent spinal studies (ED50 were always quoted in these studies) and thus this level of risk was included for comparative purposes. Lighter curves correspond to shifts at higher levels of risk. The increase in risk level used corresponds to a further shift on the curve towards the left. Thus, for each colour (blue or red) the right-hand most curve corresponds to a shift at the $1 \%$ level, whilst the lefthand curve represents a shift at the $50 \%$ level of risk of myelopathy.

Critically, all curves are bunched together illustrating that varying the risk level at which the shift occurs has little influence on the position of the final curve. However, increasing the 
conservative factor, as well as increasing the risk level at which the curves are shifted does lead to the curves spreading out further. Conversely, the curves come together at the lowest levels of risk of myelopathy, illustrating minimal influence.

This is further illustrated in Table D where values of BED, calculated from specific points of the curves in Figure D, are tabulated. The greatest difference in the tabulated values at the clinically relevant levels of risk arise from comparing the $1 \%$ and $10 \%$ levels of risk, as observed from Figure D. Further, the differences in BED values will be larger for the greater degree of conservatism and at the higher levels of risk of myelopathy. However, even in the ultra-conservative case (20\% shift) the difference in BED values between a shift at the $1 \%$ and $10 \%$ risk levels is only $101.7 \mathrm{~Gy}_{2}$ compared with $98.9 \mathrm{~Gy}_{2}$, a difference of $2.8 \mathrm{~Gy}$, or $2.75 \%$. Even for the level of risk in the rodent, $50 \%$, the comparable difference is still only $6 \%$.

Table D: Variation in the BED values of human spinal data based on the use of 54.8 Gy in 2 Gy fractions being associated with a $1 \%$ incidence of myelopathy. The associated BED values are calculated for the original non-conservative situation and for situations where there are other associated risk factors such that the BED delivered is reduced by $10 \%$ (conservative) or $20 \%$ (ultra-conservative). This conservatism is then applied at different levels of risk of myelopathy, i.e. $1 \%, 5 \%, 10 \%$ and $50 \%$, to illustrate the relative insensitivity of the change in this parameter.

\begin{tabular}{|c|c|c|c|c|c|c|c|c|c|}
\hline \multirow{3}{*}{$\begin{array}{l}\text { Risk of } \\
\text { myelopathy } \\
\text { tolerance, } R \\
(\%)\end{array}$} & \multirow{3}{*}{$\begin{array}{l}\text { For non- } \\
\text { conservative } \\
\text { policy BED } \\
\left(G_{2}\right)\end{array}$} & \multicolumn{4}{|c|}{$\begin{array}{l}\text { Conservative, } 10 \% \text { shift, } \\
\text { BED }\left(G_{2}\right)\end{array}$} & \multicolumn{4}{|c|}{$\begin{array}{l}\text { Ultra-conservative, } 20 \% \\
\text { shift, BED }\left(\mathrm{Gy}_{2}\right)\end{array}$} \\
\hline & & \multicolumn{4}{|c|}{ Risk of myelopathy (\%) } & \multicolumn{4}{|c|}{ Risk of myelopathy (\%) } \\
\hline & & $1 \%$ & $5 \%$ & $10 \%$ & $50 \%$ & $1 \%$ & $5 \%$ & $10 \%$ & $50 \%$ \\
\hline 'Tolerance'* & 100.0 & 89.0 & 88.1 & 87.6 & 85.9 & 78.1 & 76.2 & 75.3 & 71.8 \\
\hline 0.25 & 103.1 & 92.2 & 91.3 & 90.8 & 89 & 81.2 & 79.4 & 78.4 & 74.9 \\
\hline 0.5 & 106.2 & 95.3 & 94.4 & 93.9 & 92.2 & 84.3 & 82.5 & 81.5 & 78.1 \\
\hline 1.0 & 109.6 & 98.6 & 97.7 & 97.2 & 95.5 & 87.7 & 85.8 & 84.9 & 81.4 \\
\hline 2.0 & 113.3 & 102.3 & 101.4 & 100.9 & 99.2 & 91.3 & 89.5 & 88.5 & 85.1 \\
\hline 4.0 & 117.3 & 106.4 & 105.5 & 105 & 103.3 & 95.4 & 93.6 & 92.6 & 89.2 \\
\hline 6.0 & 120.0 & 109.0 & 108.1 & 107.6 & 105.9 & 98.1 & 96.2 & 95.3 & 91.8 \\
\hline 8.0 & 122.0 & 111.0 & 110.1 & 109.6 & 107.9 & 100.1 & 98.2 & 97.3 & 93.8 \\
\hline 10.0 & 123.7 & 112.7 & 111.8 & 111.3 & 109.6 & 101.7 & 99.9 & 98.9 & 95.5 \\
\hline
\end{tabular}


*as defined as $50 \mathrm{~Gy}$ in 25 fractions of $2 \mathrm{~Gy}$ in the non-conservative model: BED $100 \mathrm{~Gy}_{2}$, namely, $R=0.12 \%$.

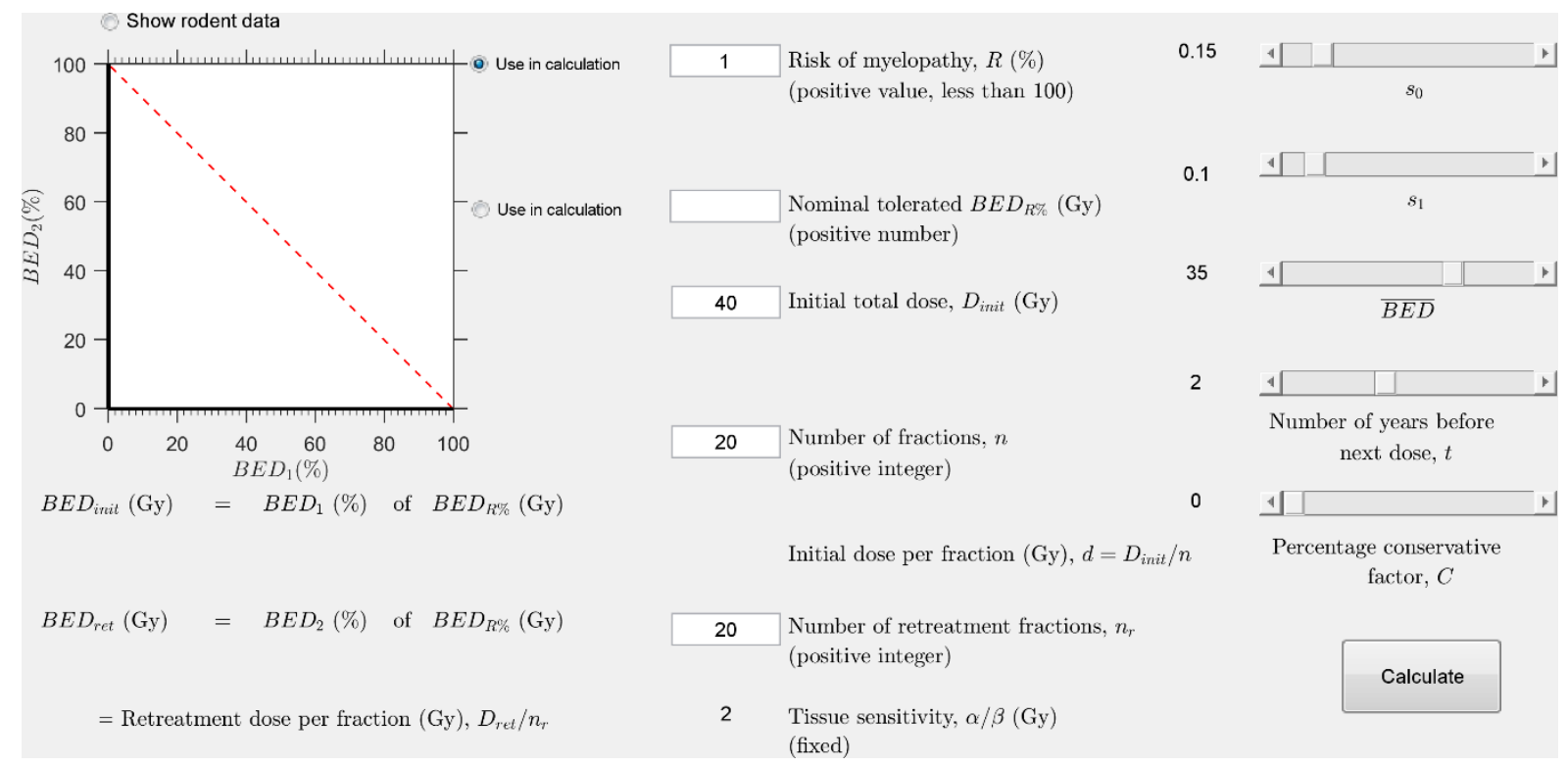

Figure B1. Default view of the BED Calculator GUI 

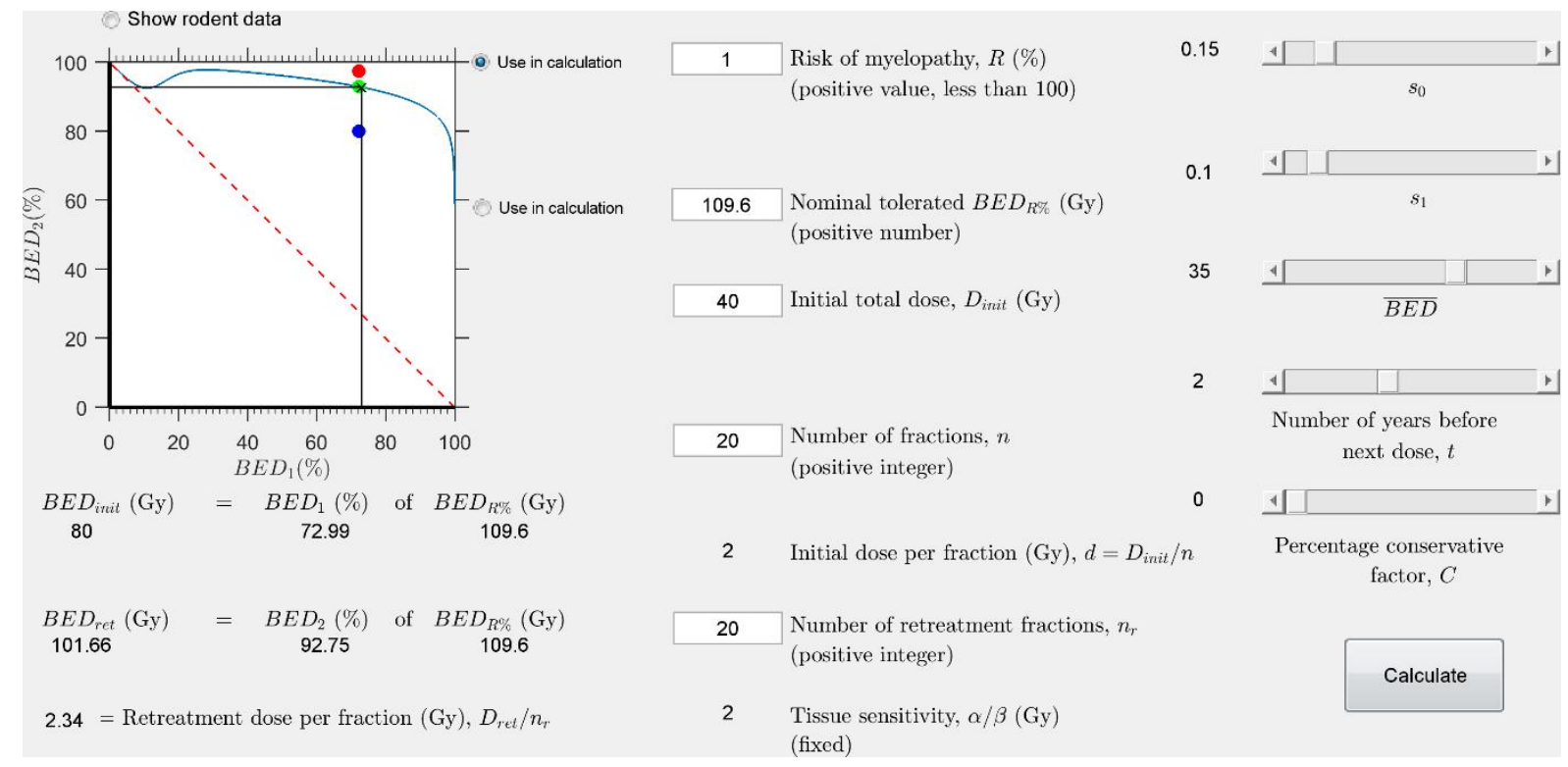

Figure B2. Pressing "Show rodent data" and "Calculate" after inserting illustrated parameters to use in the GUI produces the following output. Note that the output values match the answers presented in the non-conservative worked example. By altering the conservative factor on the right panel the GUI the user can also produce conservative estimates. For the data references, see the caption to Figure 3. 


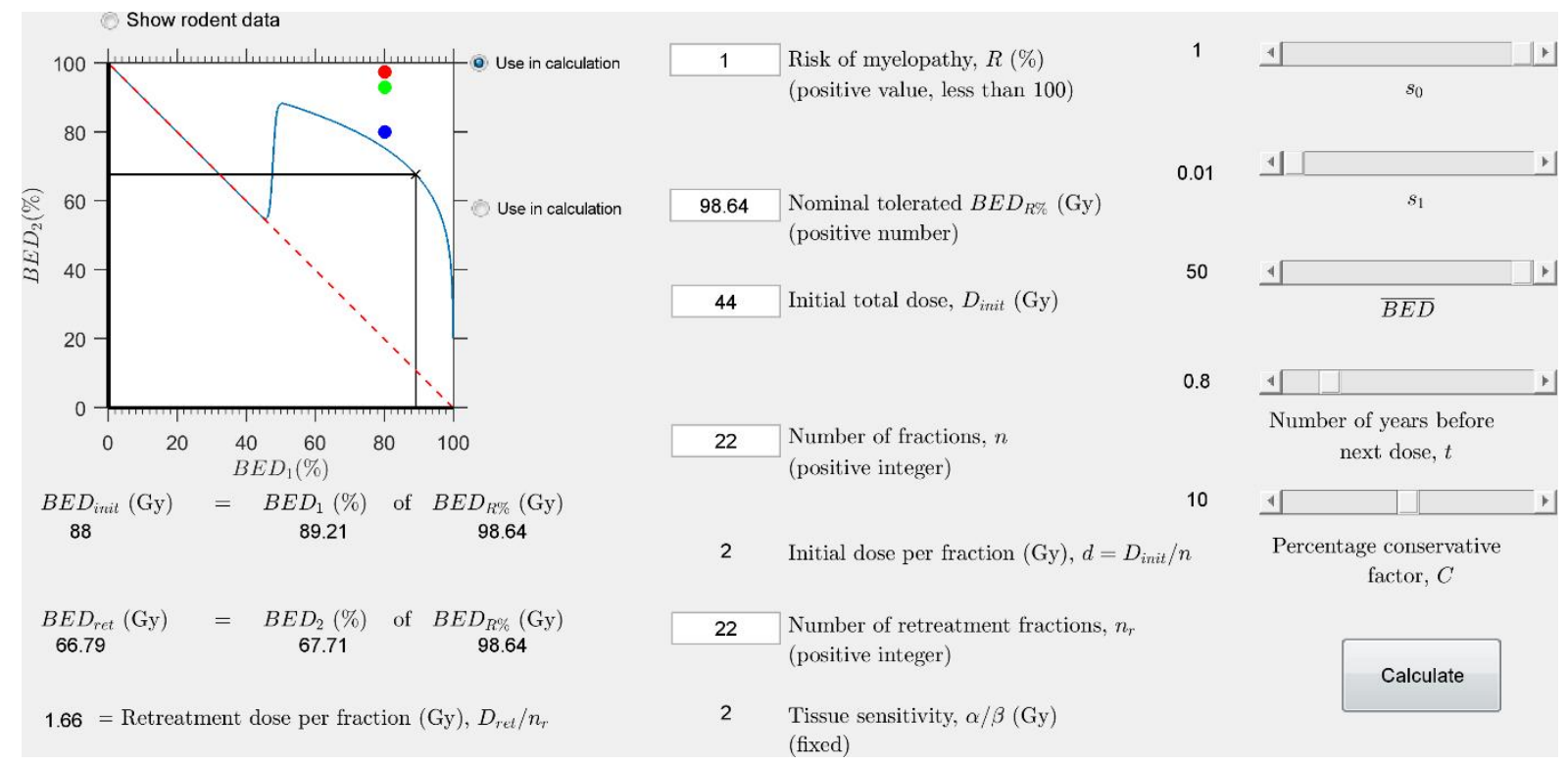

Figure B3. Update of the calculator predictions based on the illustrated non-default values. 


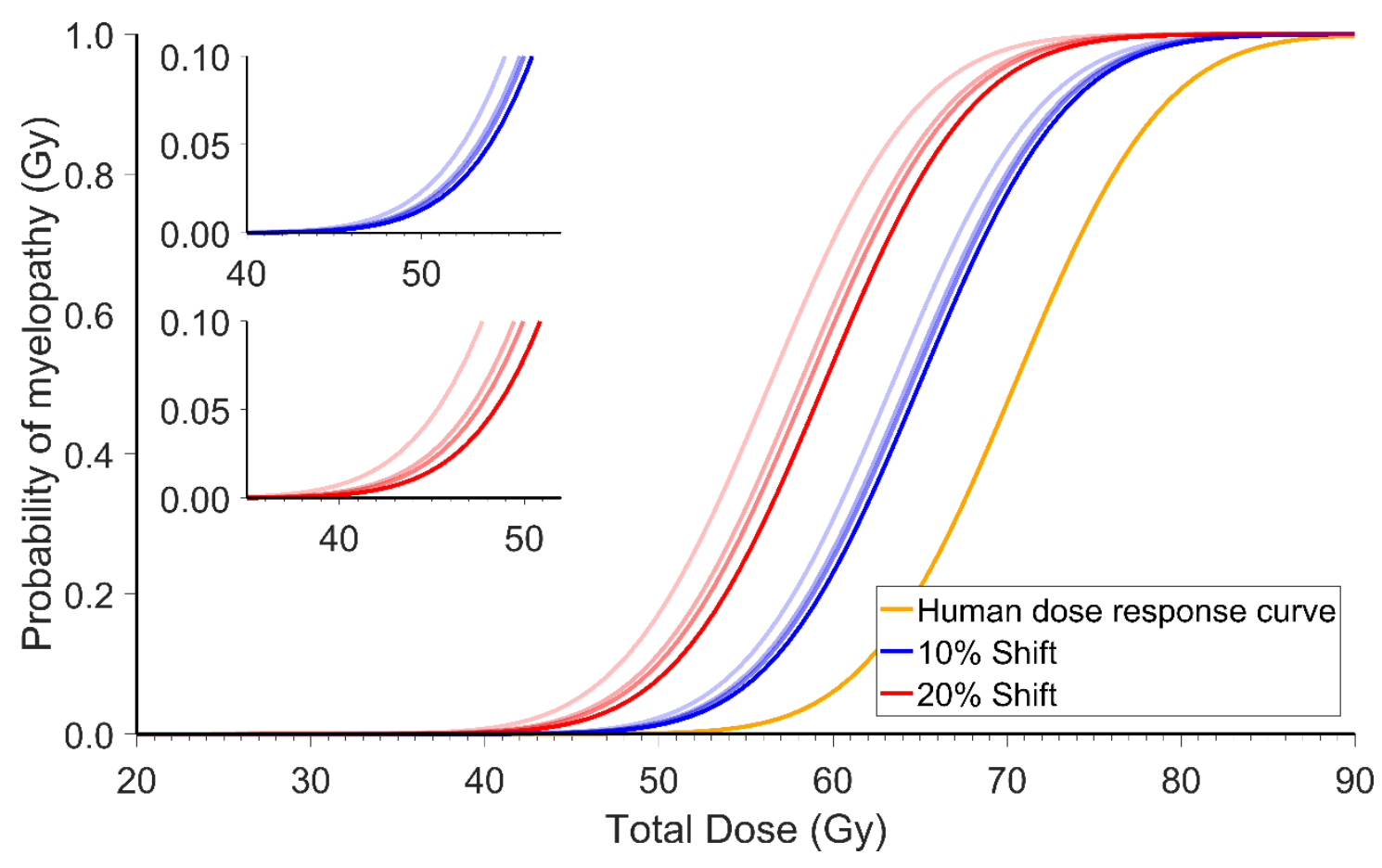

Figure D. Effect of the introduction of either conservatism or ultra-conservatism. Shifting the human non-conservative dose response curve (orange) by a 10\% (blue) and $20 \%$ (red), respectively, parallel at risk level of $1 \%, 5 \%, 10 \%$ and 50\% (progressively lighter colours, respectively). The insets are zoomed in sections of the curves in the regions of less than $10 \%$ probability of myelopathy. 